\title{
Veränderungspotenziale durch die Digitalisierung der gewerkübergreifenden Kooperation von kleinen und mittleren Unternehmen im Baugewerbe in Richtung Building Information Modeling (BIM) - Eine Fallanalyse
}

\author{
Sandra Rothenbusch ${ }^{1}$ (D) $\cdot$ Simone Kauffeld ${ }^{1}$ \\ Online publiziert: 6. August 2020 \\ (c) Der/die Autor(en) 2020
}

\section{Zusammenfassung}

In diesem Fallbeispiel der Zeitschrift „Gruppe. Interaktion. Organisation (GIO)“ wird abgeleitet, wie sich die unternehmensund gewerkübergreifende Kooperation im Baugewerbe durch die Einführung eines digitalen Tools zur 3D-Modellierung von Gebäuden verändern könnte. Die Digitalisierung durchdringt das Baugewerbe (Naegele et al. 2015) und soll im Fall von Building Information Modeling (BIM) Potenziale zur Optimierung der Planung, Fertigung und Wartung von Gebäuden bieten (z. B. Fischer et al. 2017). Im Fallbeispiel wird ein digitales Tool entwickelt, mit dem auch kleine und mittlere Unternehmen, die momentan über keine Kompetenzen und Ressourcen zur computerbasierten Modellierung von Gebäuden im mehrdimensionalen Raum verfügen, diesem Digitalisierungstrend folgen können. Zur erfolgreichen Integration eines digitalen Tools in die kooperativen Arbeitsprozesse bedarf es einer proaktiven, gemeinsamen und menschenzentrierten Betrachtung der Technologie und der Arbeitsgestaltung im Sinne des soziotechnischen Ansatzes (z. B. Parker und Grote 2020). Im Fokus des Beitrags steht die mögliche Veränderung der Arbeitsprozesse und der Arbeitsgestaltung der unternehmens- und gewerkübergreifenden Kooperation eines mittelgroßen Holzbauunternehmens in der Planungsphase des Gebäudebaus durch die Technologieeinführung. Der Ist- und Soll-Zustand sowie die möglichen Konsequenzen der Einführung des digitalen Tools werden diskutiert. Das digitale Tool verspricht eine Intensivierung der gemeinsamen Arbeit in der Planungsphase, durch die Probleme in der Ausführungsphase reduziert werden können. Neben erwünschten Veränderungen, wie den verbesserten Möglichkeiten zum Informationsaustausch und zur Abstimmung, sind vielfältige weitere Konsequenzen möglich, die durch eine frühzeitige Aufdeckung in der Entwicklungsphase eines digitalen Tools proaktiv gestaltbar werden.

Schlüsselwörter Arbeitsgestaltung · Gewerkübergreifende unternehmensübergreifende Kooperation · Building Information Modeling · Baugewerbe

Dr. Sandra Rothenbusch

s.rothenbusch@tu-braunschweig.de

1 Technische Universität Braunschweig, Braunschweig, Deutschland 


\title{
Potential for change through the digitalization of the cross-trade cooperation of small and medium sized organizations in the construction industry toward Building Information Modeling (BIM)—a case report
}

\begin{abstract}
This case report for the journal "Group. Interaction. Organization (GIO)" deduces how the cross-company and cross-trade cooperation in the construction industry could change through a digital tool for 3D modeling. Digitalization permeates the construction industry (Naegele et al. 2015) and in the case of Building Information Modeling (BIM) ought to provide potential for optimizing the planning, production and maintenance of buildings (e.g., Fischer et al. 2017). In this case study, a digital tool is being developed to enable small and medium sized organizations who currently have no competencies and resources in computer aided modeling of buildings in multidimensional space to follow this digitalization trend. For a successful integration of a digital tool in cooperative work processes, it is necessary to consider technology and work design proactively, jointly, and human centered according to the sociotechnical systems perspective (e.g., Parker und Grote 2020). The focus of this report is the possible change of work processes and work design of the cross-company and cross-trade cooperation of a medium sized wood construction company in the planning phase of building construction due to the technology implementation. The current and target state as well as the possible consequences of the technology implementation are discussed. The digital tool promises an intensification of cooperation in the planning phase so that problems in the execution phase can be reduced. Next to desirable changes, like improved possibilities for the exchange of information and coordination, several further consequences are conceivable. These will become proactively designable due to a timely recognition in the development phase of a digital tool.
\end{abstract}

Keywords Work design · Cross-company and cross-trade cooperation $\cdot$ Building information modeling $\cdot$ Construction industry

\section{Einleitung}

In der Bauwirtschaft sind knapp 850.000 Menschen in Deutschland tätig, die einen Umsatz von etwas über 50 Mrd. $€$ erwirtschaften (Statistisches Bundesamt 2019) und häufig in unternehmensübergreifenden Netzwerken beim Gebäudebau kooperieren (z.B. Chan et al. 2018; Loose 2001). Hier treffen unterschiedliche Unternehmen und Menschen aus verschiedenartigen Fachdisziplinen aufeinander, die in der Planung, Fertigung und Wartung von Gebäuden Wissen und Informationen austauschen müssen (Fischer et al. 2017). Diese Kooperationen müssen einer Kundschaft gerecht werden, die häufig durch Internetrecherchen genaue Vorstellungen über den Preis und die Qualität des gewünschten Produktes haben (Thomas 2016) und zunehmend individualisierte Wünsche äußern (z.B. Naegele et al. 2015). Zudem bevorzugen viele Kundinnen und Kunden mit Gesamtkonzepten und möglichst nur einem Ansprechpartner verstärkt Dienstleistungen ,aus einer Hand“ (z. B. Loose 2001; Naegele et al. 2015).

Potenziale zur Unterstützung der Arbeit in der Bauwirtschaft werden gegenwärtig vor allem in der Digitalisierung von Arbeitsprozessen gesehen (Fischer et al. 2017; KlemtAlbert 2018; Kocijan 2018). Beispielsweise soll mit digitalen Tools, die Building Information Modeling (BIM, z. B. Miettinen und Paavola 2014; Wu et al. 2018) ermöglichen, eine Optimierung der Kooperation in den Wertschöpfungsnetzwerken erreicht werden. So auch im vorliegenden Fall- beispiel, bei dem ein mittelgroßes Unternehmen, das auf Holzbauhäuser in den Bereichen Wohnungs-, Kommunalund Gewerbebau spezialisiert ist, betrachtet wird. Das Unternehmen übernimmt oftmals die Aufgaben eines Totalunternehmens und beauftragt Firmen zur Erbringung von Teilleistungen. Zur Optimierung der Kooperation möchte es in der Planungsphase des Gebäudebaus ein digitales Tool einsetzen, welches - orientiert an BIM - die gemeinsame Arbeit an einem 3D-Modell erlaubt.

Der vorliegende Beitrag zeigt einen Ausschnitt aus der Entwicklungsphase des digitalen Tools, in der - dem soziotechnischen Ansatz (z.B. Parker und Grote 2020; Ulich 2013) folgend - parallel zur Betrachtung technischer Aspekte, die Arbeitsgestaltung und individuellen Aspekte in den Blick genommen werden, um eine Passung zwischen technischen, organisatorischen und menschlichen Faktoren herstellen zu können. Die prädiktive Betrachtung der Arbeitsgestaltungsveränderung während der Entwicklung des digitalen Tools bietet eine wertvolle Perspektive, um die Effekte neuer Technologien beispielsweise auf die Effektivität und Effizienz (unternehmens- und gewerkübergreifender) Zusammenarbeit zu verstehen, sie für die Gestaltung guter Arbeit zu nutzen und den Weg dahin aufzuzeigen (Davis et al. 2014; Parker und Grote 2020).

Nach einleitenden Erläuterungen zur prädiktiven Betrachtung der Digitalisierung der Arbeit sowie zu Building Information Modeling, folgt eine Skizzierung des Ist-Zustands des Kooperationsprozesses und der Arbeitsgestal- 
tung in der Planungsphase des Gebäudebaus. Die geplante Gestaltung des digitalen Tools sowie die Abschätzung seiner Chancen und Risiken werden beleuchtet, um anschließend die mögliche Veränderung des Kooperationsprozesses und der Arbeitsgestaltung durch den Einsatz des digitalen Tools zu erörtern. Der Beitrag endet mit einem Fazit.

\section{Die prädiktive Betrachtung der Digitalisierung der Arbeit}

„Technology is not necessarily good, nor bad; nor is it neutral“ (Kranzberg 1986, S. 545). Die Digitalisierung der Arbeit kann beispielsweise je nach sozialer Dynamik, vorhandenen Kompetenzen der Nutzenden sowie konkreter Ausgestaltung der Technologieeinführung unterschiedliche Wirkungen auf die Arbeitsgestaltung haben (Jacobsson und Merschbrock 2018; Parker und Grote 2020). Die Arbeitsgestaltung hat wiederum Einfluss auf das Wohlbefinden, die Arbeitshaltung und die Arbeitsleistung (z.B. auf die benötigte Zeit, Kosten und Qualität) von Personen (z.B. Handke et al. 2019; Morgeson und Humphrey 2008; Parker et al. 2017). Die Auswirkungen der Digitalisierung sind aktiv gestaltbar (Sträter und Bengler 2019). Deshalb sollten so früh wie möglich - also bereits bei der Entwicklung einer Technologie - konsistent mit dem soziotechnischen Systemansatz der gemeinsamen Optimierung (joint optimization) Technologie und Arbeitsgestaltungsentscheidungen gemeinsam proaktiv und menschenzentriert betrachtet werden (Davis 2019; Davis et al. 2014; Parker und Grote 2020; Ulich 2013).

Das Scheitern von Digitalisierungsversuchen kostet Unternehmen viel Geld und verhindert die Realisierung des angestrebten Nutzens der digitalen Tools (Jasperson et al. 2005). Deshalb schlagen beispielsweise Venkatesh und Bala (2008) Maßnahmen zur Erhöhung der Technologieakzeptanz vor, die beim Design des digitalen Tools starten und sich über den gesamten Implementationsprozess erstrecken. So sollte bereits bei der Entwicklung der Technologie darauf geachtet werden, dass das digitale Tool zu den Arbeitsanforderungen und den Wertesystemen der Nutzenden passen wird. Auch Schlicher et al. (2018) betonen in ihrem Phasenmodell des Change-Managements in Digitalisierungsprozessen, dass insbesondere in der Planungsphase eine Zieldefinition und Abschätzung der Konsequenzen der Technologieimplementierung für die Stakeholder für den Erfolg der Technikimplementierung relevant sind.

Im Folgenden wird ein Überblick über Building Information Modeling gegeben, um anschließend auf die Ziele und angenommenen Konsequenzen des digitalen Tools eingehen zu können.

\section{Möglichkeiten des Building Information Modelings (BIM) zur Unterstützung von Kooperationen im Baugewerbe}

Unter dem Schlagwort Building Information Modeling (BIM) wird die Nutzung virtueller, mehrdimensionaler Repräsentationen für die Planung, Ausführung und Nutzung von Gebäuden propagiert (Miettinen und Paavola 2014). BIM ermöglicht als Informations- und Kommunikationstechnologie (IKT) die interdisziplinäre Zusammenarbeit über den gesamten Projektzyklus hinweg (Chan et al. 2018). Allerdings handelt es sich bei BIM keineswegs nur um eine Software-Lösung, sondern um eine neue Form der Zusammenarbeit, bei der der Faktor „Mensch“ entscheidend ist (Klemt-Albert 2018) und durch die Rollenveränderungen bei Kundinnen und Kunden, Architektinnen und Architekten, Ingenieurinnen und Ingenieuren sowie Bauunternehmerinnen und -unternehmern (Sebastian 2011; Bråthen und Moum 2016) hervorgerufen werden können. Der Einfluss und damit das Potenzial von BIM in der gewerkübergreifenden Kooperation im Baugewerbe bezieht sich auf drei Ebenen (Jacobsson und Merschbrock 2018): Erstens, als technisches Produkt bietet BIM den Vorteil präziser, geometrischer Gebäuderepräsentationen in einer integrierten Datenumgebung. BIM kann jedoch, zweitens, auch als ein Prozess der Entwicklung und Nutzung digitaler Repräsentationen und somit als integraler Bestandteil der Projektentwicklung, des Projektmanagements und des Ablieferungsprozesses (Bryde et al. 2013) verstanden werden. Aus diesem Blickwinkel steuert BIM sowohl die Möglichkeit der schnellen und akkuraten Aktualisierung bei Veränderungen (Manning und Messner 2008) als auch die Unterstützung bei der Kostenreduktion und Kontrolle im Projektverlauf (Bryde et al. 2013) bei. BIM als IKT beeinflusst, drittens, aus der Systemperspektive das Geschäftsund Kommunikationsnetzwerk und beachtet kontextuelle Rahmenbedingungen. Das Potenzial von BIM wird aus der Systemperspektive vor allem in der erhöhten Kommunikation zwischen allen Projektbeteiligten gesehen. Für eine umfassende Integration von BIM in Projekte des Baugewerbes siehe beispielsweise Integrating Project Delivery (IPD; Fischer et al. 2017).

Die Vorteile von BIM werden nicht nur in einer besseren Planbarkeit eines Bauprojekts, der konsistenteren Dokumentation und der vereinfachten Koordination gesehen, sondern auch in der Erhöhung der Entscheidungssicherheit bei Kundinnen und Kunden und der Möglichkeit, den Gebäudebesitzerinnen und -besitzern wertvolle Informationen sinnvoll aufbereitet übergeben zu können (Crotty 2012). Insgesamt schlussfolgern Forgues et al. (2016), dass BIM die Produktivität und die Effizienz erhöht und das Endprodukt verbessert. 
Der reine Einsatz einer BIM-orientierten, technischen Lösung, führt nicht automatisch zu einer Verbesserung der Kooperation, da sie unter anderem in Normen, Referenzrahmen, Regeln, Regularien, Unternehmenskulturen und Merkmalen des Wirtschaftszweiges situiert ist (Linderoth 2010). Beispielsweise ist die Nutzung von 2D-Modellen im Baugewerbe kulturell stark verankert, sodass der Wechsel auf eine dreidimensionale Modellierung auf Widerstand stoßen kann (Babič und Rebolj 2016). Die Kooperationsbeteiligten müssen ihre Beziehungen selbst so anpassen, dass durch BIM die Kooperation verbessert werden kann (Forgues et al. 2016). Vor allem im Baugewerbe, in dem die Gewerke bislang relativ isoliert und unabhängig arbeiten, müssen daher die sozialen und menschlichen Bedingungen ausreichend für eine erfolgreiche Implementierung mitbedacht werden (Forgues et al. 2016; Miettinen und Paavola 2014; Neff et al. 2010). Zudem scheint BIM seine Vorteile nur zu entfalten, wenn der digitale Informationsstand mit dem realen Konstruktionsprozess durchgehend synchronisiert ist (Chen et al. 2015).

Die technologischen Visionen um BIM tendieren dazu, utopisch zu sein (Miettinen und Paavola 2014), weshalb teilweise zu hohe Erwartungen gestellt werden, die in der Realität nicht umsetzbar sind und zu Enttäuschungen führen können (Borup et al. 2006). In der Praxis sind daher hybride Formen von BIM nicht ungewöhnlich (Davies et al. 2017). Sie werden unter anderem benötigt, um kleinen und mittleren Unternehmen die Teilhabe an diesem Digitalisierungstrend zu ermöglichen, vor allem wenn diese Unternehmen nicht über die Kompetenzen und Ressourcen zur CAD(Computer Aided Design-)basierten 3D-Modellierung sowie über BIM-Koordinatorinnen und -koordinatoren verfügen. Zur Relevanz der Koordinationsrolle bei BIM siehe Jacobsson und Merschbrock (2018).

Um die Konsequenzen eines BIM-orientierten digitalen Tools für die Arbeitsgestaltung in der Praxis zu erörtern, wird im Folgenden die gewerkübergreifende Kooperation im Baugewerbe an einem Fallbeispiel dargestellt.

\section{Skizzierung der gewerkübergreifenden Kooperation im Baugewerbe}

Im Fallbeispiel wird die gewerkübergreifende Kooperation eines mittelgroßen Holzbauunternehmens mit rund 150 Mitarbeitenden bei Bauprojekten - wie beispielsweise dem Bau eines Einfamilienhauses - betrachtet. Für die Darstellung des Fallbeispiels werden folgende Informationsquellen genutzt: Erstens, um Informationen zum Kooperationsprozess, zu typischen Fehlern in der Kooperation und zu den Anforderungen des digitalen Tools zu erhalten, wurde ein Workshop mit Vertreterinnen und Vertretern des Holzbauunternehmens und seiner Kooperationsbeteiligten aus dem HSLE-Bereich (Heizung, Sanitär, Lüftung, Elektro) durchgeführt. Zusätzlich wurden die Informationen des Workshops vom Holzbauunternehmen in einem Dokument zusammengefasst und um Hinweise aus weiteren Besprechungen mit den Kooperationsbeteiligten ergänzt. Zweitens wurde eine Sammlung von möglichen Vor- und Nachteilen des digitalen Tools erarbeitet. Dafür trugen Vertreterinnen und Vertretern des Holzbauunternehmens, des an der Entwicklung des digitalen Tools beteiligten Softwareunternehmens und eines Weiterbildungsinstituts im Handwerk ( $n=4$ Personen) im ersten Schritt während eines gemeinsamen Treffens Vor- und Nachteile zusammen. Zur genaueren Analyse und Ergänzung der bereits genannten Vor- und Nachteile wurden vier bis fünf Monate später vertiefende teilstrukturierte Einzelinterviews ( $n=4$ Interviews) durchgeführt. Zum Zeitpunkt der Datenerhebung lag die Konzeption des digitalen Tools vor, jedoch noch kein Prototyp. Im Folgenden werden zuerst schematisch die Kooperationsart sowie der -prozess erläutert und anschließend typische Problemfelder adressiert.

\subsection{Kooperationsart und -prozess}

Das Holzbauunternehmen übernimmt in der Kooperation mit Unternehmen aus dem HSLE-Bereich neben der Herstellung von Holzbau-Elementen häufig die Funktion des Totalunternehmens. Die Unternehmen in der Kooperation gehören zu aufeinanderfolgenden Wirtschaftsebenen in der Wertschöpfungskette und gehen mit komplementären Kompetenzen eine so genannte vertikale Kooperation ein (Kategorisierung siehe Friese 1998). Sie stellt eine Form der interdisziplinären Zusammenarbeit dar, da „Personen unterschiedlicher fachlicher, funktioneller oder disziplinärer Hintergründe gemeinsam an einem Produkt oder einer Fragestellung arbeiten“ (Brandstädter et al. 2018, S. 36). Insgesamt handelt es sich bei der vorliegenden Kooperation mit einem Total- und mehreren Subunternehmen um eine klassische Form der Zusammenarbeit im Baugewerbe (Jones und Lichtenstein 2009; Klemt-Albert 2018), die zu den präferierten Formen der Zusammenarbeit in dem Bereich zählt (Eccles 1981).

Der vom Holzbauunternehmen beschriebene Kooperationsprozess wurde orientiert an der Business Process Modelling Notation (BPMN; z. B. Aagesen und Krogstie 2015) schematisch in Abb. 1 zusammengefasst. In der Planungsphase erstellt das Holzbauunternehmen - aufbauend auf Plänen des Architekturbüros - einen detaillierten Plan, der um die wesentlichen Randbedingungen von der Statikerin oder dem Statiker ergänzt werden. Anschließend werden die Gewerkplanungen der Elektro- sowie Sanitär-, Heizungsund Lüftungsbetriebe eingebunden. Die Abstimmung mit dem Tief- und Massivbau erfolgt meistens nachgeschaltet. Zur Planung vieler Bauteile sind Informationen aus ande- 


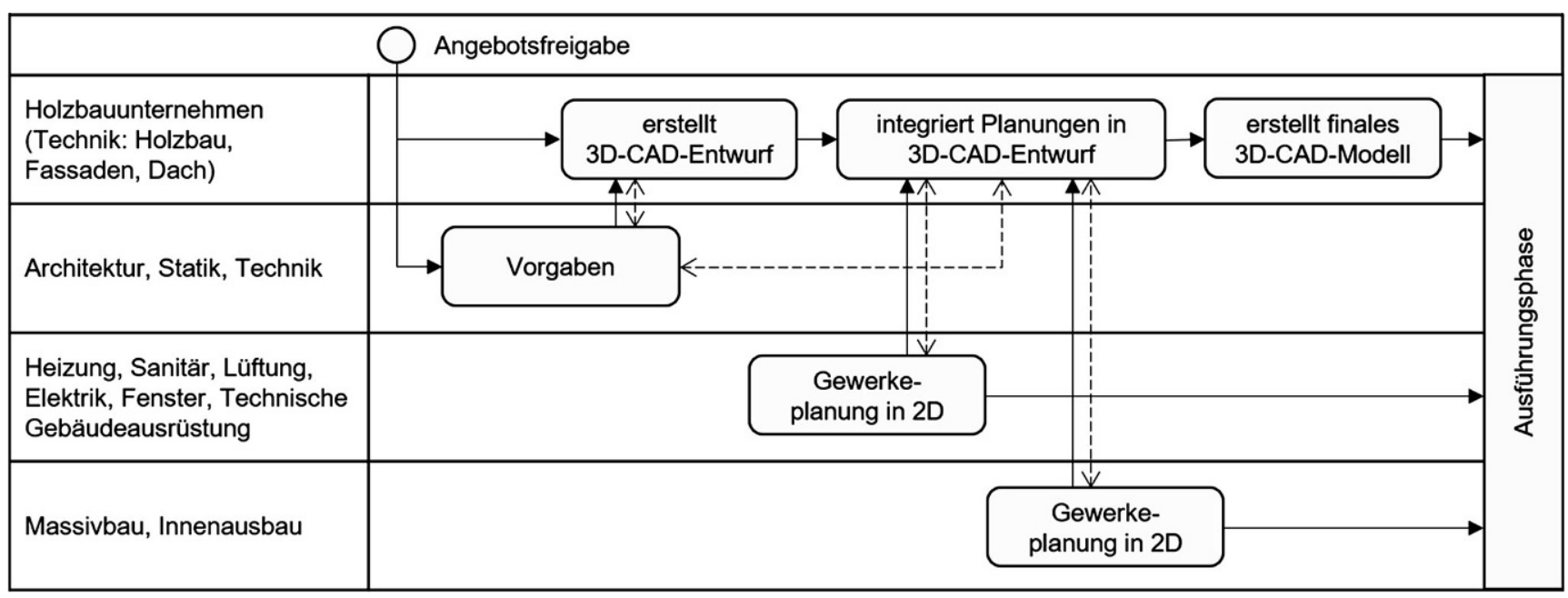

Abb. 1 Prozess der im Fallbeispiel geschilderten gewerkübergreifenden Kooperation in der Planungsphase im Baugewerbe. Die durchgehenden Pfeile stellen die standardisierte Informationslieferkette dar. Die gestrichelten beidseitigen Pfeile symbolisieren bedarfsbedingte Nachfrageschleifen

ren Gewerken zu berücksichtigen (z.B. Abhängigkeit der Elektroinstallationen von der Sanitärplanung). Die Planung dieser Schnittmengen erfordert im Prozess oft iterative Klärungen mit den Kooperationsbeteiligten, die vornehmlich über das Holzbauunternehmen laufen. Im Gegensatz zu vergleichbaren Kooperationen im Bauhandwerk werden nach Angabe des Holzbauunternehmens bereits früher im Prozess eineindeutige Informationen aus den anderen Gewerken benötigt (z.B. zu Steckdosen, Sicherheitstechnik und Durchbrüchen für Rohre), um die Holzwände planen und automatisiert fertigen zu können.

\subsection{Problemfelder in der Kooperation}

Dieser Kooperationsprozess kann jedoch durch unterschiedliche Probleme behindert werden. Das Holzbauunternehmen, welches in dieses Projekt eingebunden war, beschrieb zwei konkrete Ereignisse, die prototypisch für häufiger auftretende Probleme in der Planungsphase stehen: Beispielsweise lieferte ein Sanitärunternehmen einen Grundriss zur Planung der für die Sanitärbauteile benötigten Durchbrüche an den Holzwänden an das Holzbauunternehmen. Allerdings fehlten viele Angaben, etwa die Höhenangaben zu den Durchbrüchen und die Angaben zur Form der Durchbrüche (rund oder eckig). In einem weiteren Beispiel fiel erst während der Fertigung der Holzelemente auf, dass es bei der vorliegenden Bauplanung zu einer Kollision zwischen Badentlüfter, Badewanne und Dachfenster kommen würde. Es stellte sich heraus, dass das Sanitärunternehmen den Grundriss der Holzkonstruktion nicht ausreichend beachtete und die Position des Entlüfters nicht vermerkte. Zudem wurden die Änderungswünsche des Kunden nicht an das Holzbauunternehmen weitergeleitet.
Die Sammlung der typischen Probleme für die gemeinsame Aufgabe der Gebäudeplanung wurde anhand des Mensch-Technik-Organisation-Dreiecks (Ulich 2013) kategorisiert (siehe Abb. 2). Die meisten typischen Probleme befinden sich in der Schnittmenge zwischen Mensch und Organisation. Die Kooperationsbeteiligten liefern teilweise unzureichende Informationen, es kommt im Kooperationsprozess häufig zu unabgestimmten Änderungen, die Arbeitsprozesse verzögern sich, Verantwortlichkeiten sind nicht immer geklärt und zusätzliche Arbeitsschleifen müssen eingebaut werden, weil die einzelnen Gewerkplanungen nicht miteinander kompatibel sind. Zudem wird bezüglich der Organisation bemängelt, dass vor allem bei kurzfristigen Änderungen die Dokumentation schwerfällt.

In Bezug auf die Technik und ihre Schnittmengen zur Organisation und zum Menschen können drei typische Problemfelder ausgemacht werden (siehe Abb. 2). Zum einen ist der Digitalisierungsgrad innerhalb der Kooperationen heterogen. Der Unterscheidung von Lichtblau et al. (2018) folgend, kann das Holzbauunternehmen als digitalisiert bezeichnet werden, da ihre Unternehmensprodukte virtualisiert sind. Die Planung der Holzbau-Elemente erfolgt mittels CAD-Modellen, die eine automatisierte Produktion in der unternehmenseigenen Werkhalle ermöglichen. Die anderen Unternehmen sind vornehmlich der computerisierten Kategorie zuzuordnen, die zwar auch IKT und das Internet nutzen, jedoch keine virtualisierten Prozesse und Produkte haben. Sie nutzen im Gegensatz zum Holzbauunternehmen, das vorrangig mit 3D-Modellen arbeitet, noch bevorzugt 2D-Pläne zur Gewerkplanung. Dieser Technikbruch zwischen 2D- und 3D-Modellierung wurde als bedeutsame Fehlerquelle identifiziert, da die 2D-Planung das Aufdecken von Fehlern aufgrund des geringeren Informationsumfangs 
Abb. 2 Kategorisierung der Problemfelder nach Mensch, Technik und Organisation (Ulich 2013)

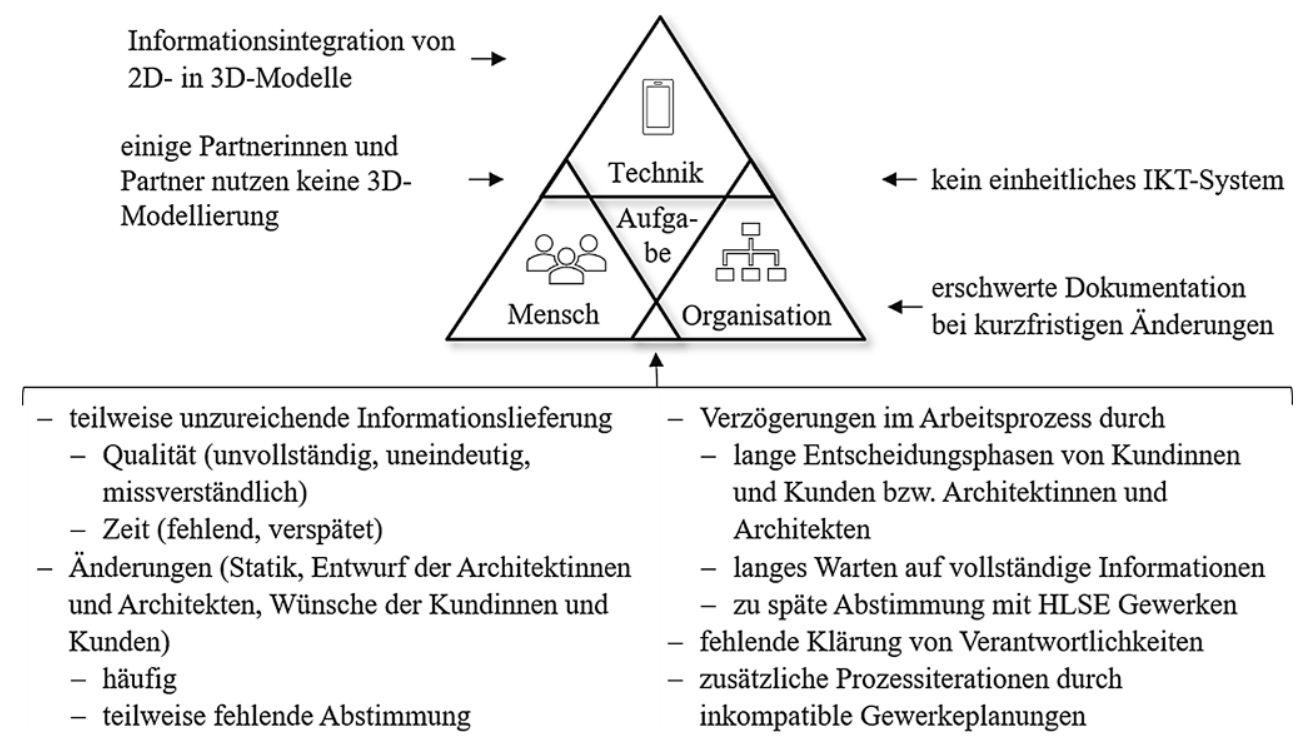

Informationsintegration von artner nutzen keine 3D Modellierung

- Verzögerungen im Arbeitsprozess durch und Kunden bzw. Architektinnen und Architekten

- langes Warten auf vollständige Informationen

- zu späte Abstimmung mit HLSE Gewerke

- zusätzliche Prozessiterationen durch nompatible Gewerkeplanungen 
Tab. 1 Ist-Zustand der Arbeitsgestaltung und mögliche Veränderungen durch das digitale Tool

\begin{tabular}{|c|c|c|c|}
\hline \multicolumn{2}{|c|}{ Arbeitsgestaltungsdimension } & \multirow{2}{*}{$\begin{array}{l}\text { Ist-Zustand der Planungsphase im Gebäudebau } \\
\text { Sequenzielle, teilweise reziproke Arbeitsweise }\end{array}$} & \multirow{2}{*}{$\begin{array}{l}\text { Mögliche Änderungen durch das digitale Tool } \\
\checkmark \text { Verbesserte Koordination }\end{array}$} \\
\hline Interdependenz & Aufgaben- & & \\
\hline & $\begin{array}{l}\text { interdepen- } \\
\text { denz }\end{array}$ & Koordination teilweise eingeschränkt & $\begin{array}{l}\oslash \text { Abweichung von der sequenziellen Arbeitswei- } \\
\text { se (stärkere Reziprozität) }\end{array}$ \\
\hline & & & ? Interdependenz sichtbarer \\
\hline & $\begin{array}{l}\text { Ergebnis- } \\
\text { interdepen- }\end{array}$ & $\begin{array}{l}\text { Gebäudeplan hauptsächlich als Ergebnis vieler } \\
\text { Einzelleistungen aufgefasst, belohnt und }\end{array}$ & $\begin{array}{l}\checkmark \text { Vereinfachte Identifizierung und Beurteilung } \\
\text { der Einzelleistungen }\end{array}$ \\
\hline & & kommuniziert & $\begin{array}{l}\text { ? Erhöhung der Wahrnehmung und Kommunikati- } \\
\text { on des Gebäudeplans als Gruppenleistung }\end{array}$ \\
\hline \multirow[t]{5}{*}{$\begin{array}{l}\text { Wissens- } \\
\text { merkmale }\end{array}$} & $\begin{array}{l}\text { Aufgaben- } \\
\text { unsicherheit }\end{array}$ & $\begin{array}{l}\text { Veränderungen von Kundenwünschen wahrscheinlich } \\
\text { Sicherheit über Aufgabenziele }\end{array}$ & $\begin{array}{l}\text { ? Schnellere Benachrichtigung und Abstimmung } \\
\text { bei unvorhergesehenen Ereignissen }\end{array}$ \\
\hline & & $\begin{array}{l}\text { Berechenbarkeit der exakten Zielerreichung kann } \\
\text { eingeschränkt sein }\end{array}$ & ? (Anfangs) unberechenbarer \\
\hline & & & ? Dynamischer \\
\hline & $\begin{array}{l}\text { Aufgaben- } \\
\text { routine }\end{array}$ & $\begin{array}{l}\text { Repetitive Aufgabe } \\
\text { - viele Probleme, Prozesse und Lösungen bekannt } \\
\text { - Etablierung vieler Routinen }\end{array}$ & $\begin{array}{l}\text { ? Anfangs Erhöhung der Neuartigkeit durch unbe- } \\
\text { kannte Prozesse rund um die Nutzung des digitalen } \\
\text { Tools }\end{array}$ \\
\hline & & $\begin{array}{l}\text { Teilweise ineffektive Routinen bei typischen Proble- } \\
\text { men in der Zusammenarbeit }\end{array}$ & $\begin{array}{l}\text { ? Aufbau effektiver Routinen für typische } \\
\text { Probleme }\end{array}$ \\
\hline
\end{tabular}

Teilweise Komplikationen durch unterschiedliche Reaktion der beteiligten Unternehmen auf neuartige Entwicklungen

$\begin{array}{ll}\begin{array}{ll}\text { Aufgaben- } \\ \text { komplexität }\end{array} & \begin{array}{l}\text { Komplex } \\ \text { Gut strukturiert } \\ \text { Niedrige bis mittlere Aufgabenmehrdeu }\end{array} \\ \begin{array}{l}\text { Problem- } \\ \text { lösung }\end{array} & \text { Hauptsächlich intellektuelle Aufgaben }\end{array}$

Informations- Hohes Ausmaß an Informationsbeschaffung und verarbeitung -verarbeitung

Beeinträchtigung durch teilweise mangelhafte Informationsqualität und ungenügende Abstimmungen

Arbeits- $\quad$ Rollen- $\quad$ Im unteren Bereich für gewerkspezifische Aufgaben bezogene ambiguität

Anfor-

derungen

Arbeits-

bezogene

Ressourcen

Zeitdruck

Häufig straffer Zeitplan

Vorhandener Zeitdruck führt in Kombination mit Problemen zu Stress, unstrukturierter IKT-Nutzung und unvollständiger Dokumentation

Erhöhte Rollenambiguität im interdisziplinären gewerkübergreifenden Austausch
Teamautonomie Hauptsächlich Orientierung an system-definierten linearen Interaktionsprozeduren

Feedback

Soziale Unterstützung
Feedback v. a. bei Problemen

Abschlussmeetings mit langjährigen Partnern

Hauptsächlich einfaches Netzwerk mit bilateralen Unterstützungsmöglichkeiten zwischen Totalunternehmen und Subunternehmen
Verbesserter Umgang mit Aufgabenkomplexität

? Reduktion der Mehrdeutigkeit

? (Anfänglich) höhere Komplexität durch neue Arbeitsprozesse

? Besserer Umgang mit dem Grad an Problemlöseanforderungen

? Eingeschränkter Diskussionsraum

$\checkmark$ Besserer Umgang mit Grad an Informationsbeschaffung und -verarbeitung

? Zunahme der Informationsverarbeitungsgrades für Subunternehmen

$\checkmark$ Klarere Verhaltensanweisungen für den interdisziplinären Austausch

? Anfänglich Verringerung der Rollenklarheit durch Veränderung des Arbeitsprozesses

? Aufbau mentaler Modelle für die gewerkübergreifende Kooperation

? Aufbau von T-Kompetenzen

$\checkmark$ Mehr Möglichkeiten zum effizienten Umgang mit Zeitdruck (v. a. der durch Probleme ausgelöst wird)

? Erhöhung der Teamautonomie durch vermehrt parallele und reziproke Prozesse

$\checkmark$ Systembedingtes + schnelleres Feedback

? Höhere Häufigkeit

? Unabhängiger von Problemen

? Erhöhung der Identifikation als Team und der

Presence Awareness

? Komplexeres Netzwerk mit multilateralen Unterstützungsmöglichkeiten zwischen allen Kooperationsbeteiligten

$\checkmark$ Veränderung durch Holzbauunternehmen gewünscht, $\oslash$ Veränderung durch Holzbauunternehmen nicht gewünscht, ? keine Angabe zur Erwünschtheit der Veränderung durch die Kooperationsbeteiligten 
Ergebnisinterdependenz. Die Ergebnisinterdependenz beschreibt, inwiefern das Ergebnis der Arbeit eher als Gruppenleistung anstatt als Ergebnis von individuellen Einzelleistungen aufgefasst, belohnt und kommuniziert wird (Courtright et al. 2015). Im Fallbeispiel wird das Ergebnis der Planungsphase (also das fertig geplante Gebäude) laut dem Holzbauunternehmen tendenziell als Ergebnis vieler Einzelleistungen aufgefasst, belohnt und kommuniziert. Beispielsweise sind die Subunternehmen monetär über vertragliche Regelungen einzeln an das Totalunternehmen gekoppelt. In einzelnen Fällen wird das Gesamtergebnis mit (potenziell) langjährigen Partnerinnen und Partnern in einer Abschlussbesprechung betrachtet, sodass die Wahrnehmung des Gebäudeplans als Gruppenleistung gegebenenfalls erhöht wird. Es liegen keine Äußerungen vom Holzbauunternehmen vor, die sich auf einen Änderungswunsch bezüglich der Ergebnisinterdependenz beziehen. Allerdings soll durch das digitale Tool die Arbeit der einzelnen Kooperationsbeteiligten besser dokumentiert werden, sodass bei auftretenden Problemen die Fehlerquelle effizienter aufgedeckt werden kann.

\subsection{Wissensmerkmale}

Wissensmerkmale beziehen sich auf wissensbezogene Aspekte der Arbeit und umfassen die Aufgabenunsicherheit, -routine und -komplexität sowie das involvierte Ausmaß an Problemlösen und Informationsverarbeitung (Morgeson und Humphrey 2006).

Aufgabenunsicherheit. Die Arbeitsgestaltungsdimension Aufgabenunsicherheit beschreibt, wie unvorhersehbar und dynamisch die Aufgabenumgebung eines Teams ist (Cordery et al. 2010) und inwiefern dadurch Unsicherheit in Bezug auf die Aufgabenziele und ihre Erreichung entstehen (Painter et al. 2016). Insbesondere die Änderungen von Kundenwünschen, die zu allen Zeitpunkten der Kooperation möglich sind, können unvorhersehbare Ereignisse hervorrufen. Obwohl hieraus keine Unsicherheit in Bezug auf die Aufgabenziele resultiert, kann die exakte Zielerreichung je nach spezifischem Bauprojekt weniger sicher bzw. berechenbar sein. Die Kooperationsbeteiligten äußerten sich nicht direkt zur Änderung der Aufgabensicherheit durch das digitale Tool.

Aufgabenroutine. Die Aufgabenroutine bezieht sich auf das Ausmaß der Neuartigkeit von Prozessen, Problemen oder angestrebten Lösungen, mit denen Teams konfrontiert werden (Malhotra und Majchrzak 2014). Da es sich beim Hausbau um eine repetitive Aufgabe handelt, konnten sich diesbezüglich viele Routinen etablieren. Allerdings treten beispielsweise durch die stetige Weiterentwicklung im Baugewerbe und die immer stärker individualisierten Kunden- wünsche neuartige Probleme, Prozesse und Lösungsideen auf. Die kooperierenden Unternehmen reagieren unterschiedlich schnell auf Neuerungen, wie beispielsweise auf die Verbreitung von 3D-Modellierung im Baugewerbe, wodurch es zu Komplikationen in der Zusammenarbeit kommen kann. Es wurden allerdings keine Änderungswünsche bezüglich der Aufgabenroutine genannt.

Aufgabenkomplexität. Aufgaben mit einer hohen Aufgabenkomplexität sind komplex, unstrukturiert und mehrdeutig (McGrath 1984). Die Planung eines Gebäudes ist eine komplexe Aufgabe, die je nach Gebäudetyp (z.B. Einfamilienhaus versus Seniorenheim) und Anforderungen (z. B. Rohbau oder schlüsselfertige Übergabe) an Komplexität dazugewinnt. Jedoch ist die Aufgabe vor allem durch die Etablierung von Routinen insgesamt gut strukturiert. Die Aufgabenmehrdeutigkeit kann nach der Definition von Fiedler (1967) als niedrig bis mittel beurteilt werden: Das Aufgabenziel ist meistens klar, die Anzahl an Wegen zum Ziel und korrekten Lösungen oftmals überschaubar und die Lösungen normalerweise überprüfbar. Das Holzbauunternehmen erhofft sich durch das digitale Tool einen verbesserten Umgang mit der Aufgabenkomplexität.

Problemlösung. Die Work Design-Dimension Problemlösung erfasst, inwiefern einzigartige Ideen und Lösungen während der Arbeit benötigt werden (Jackson et al. 1993; Wall et al. 1990). Unterschieden werden können intellektuelle Aufgaben, zu denen korrekte Lösungen gefunden werden müssen (hohe Problemlöseanforderungen) und Beurteilungsaufgaben, die das Treffen von Urteilen und Einschätzungen beinhalten (niedrige Problemlöseanforderungen; Laughlin und Ellis 1986). In der Kooperation bei der Hausbauplanung liegt der Schwerpunkt auf intellektuellen Aufgaben, für die korrekte Lösungen gefunden werden müssen. Es liegen keine Änderungswünsche zu dieser Dimension vor.

Informationsverarbeitung. Die Informationsverarbeitung beschreibt das Ausmaß, in dem eine Aufgabe das Besorgen und Verarbeiten von Informationen verlangt (Morgeson und Humphrey 2006). Für eine erfolgreiche Integration der Gewerkplanungen zu einem Gebäudeplan wird ein hohes Ausmaß an Informationsbeschaffung und -verarbeitung benötigt. Dieser Bereich kann jedoch aufgrund der teilweise mangelhaften Informationsqualität und oftmals ungenügenden Abstimmung beeinträchtigt sein. Das Holzbauunternehmen erhofft sich durch die Nutzung des digitalen Tools einen verbesserten Umgang mit dem hohen Ausmaß an Informationsbeschaffung und -verarbeitung. 


\subsection{Arbeitsbezogene Anforderungen}

Unter arbeitsbezogenen Anforderungen werden physische, psychische, soziale und organisationale Aspekte einer Arbeit zusammengefasst, die anhaltende physische und/oder psychische Anstrengung benötigen und dadurch mit bestimmten physischen und/oder psychischen Kosten verbunden sind (Bakker und Demerouti 2007). Hierunter fallen die arbeitsbezogene Rollenambiguität sowie der Zeitdruck (Handke et al. 2020).

Rollenambiguität. Die Rollenambiguität beschreibt das Ausmaß, in dem eine Arbeit keine klare Anleitung zu erwarteten Rollen und Verhaltensweisen liefern kann (Kahn et al. 1964). Der Arbeitsprozess der vorliegenden Kooperation ist insgesamt gut strukturiert und bietet vor allem gewerkspezifische Richtlinien und Anweisungen zur Rolle und zu den Verhaltensweisen der Kooperationsbeteiligten. Allerdings weisen die Aussagen des Holzbauunternehmens auf eine Rollenambiguität in Bezug auf den interdisziplinären, gewerkübergreifenden (vor allem unternehmensübergreifenden) Austausch hin. Der Wissensaufbau, wie konkret zusammengearbeitet wird und welche Probleme typisch in der Interaktion, beispielsweise zwischen dem Sanitärund Holzbauunternehmen, sind, erfolgt eher erfahrungsbasiert. Die Erwartungen an Rollen und Verhaltensweisen bei gewerkübergreifenden Aufgaben werden eher implizit vermittelt oder erst dann geäußert, wenn Probleme zwischen Gewerken auftreten. Die Rollenambiguität könnte zudem dadurch verstärkt werden, dass die Beteiligten jeweils über ein anderes Set an fachspezifischen Kompetenzen und gegebenenfalls nicht über ausreichend Wissen aus den anderen Fachbereichen verfügen. Die Änderungswünsche des Holzbauunternehmens durch das digitale Tool weisen auf eine erhoffte Reduzierung der Rollenambiguität hin. Durch das digitale Tool sollen klare Anweisungen gegeben werden, welche Informationen relevant sind.

Zeitdruck. Insgesamt wird häufig ein straffer Zeitplan verfolgt, um den Kundinnen und Kunden Gebäude schnellstmöglich übergeben zu können. Aufgrund des straffen Zeitplans führen die geschilderten typischen Probleme häufig zu Stress. Sie bringen den Zeitplan in Gefahr und lösen eine unstrukturierte IKT-Nutzung sowie unvollständige Dokumentation aus. Durch die Nutzung des digitalen Tools wird nicht angestrebt, den Zeitdruck per se zu verändern, jedoch einen verbesserten Umgang damit, vor allem wenn Probleme auftreten, zu erreichen.

\subsection{Arbeitsbezogene Ressourcen}

Unter arbeitsbezogenen Ressourcen werden physische, psychische, soziale und organisationale Aspekte der Arbeit zu- sammengefasst, die beim Erreichen von Arbeitszielen helfen, arbeitsbezogene Anforderungen zu verringern und/oder die persönliche Weiterentwicklung zu unterstützen (Demerouti et al. 2001). Zu den arbeitsbezogenen Ressourcen zählen die Teamautonomie, das Feedback und die soziale Unterstützung (Handke et al. 2020).

Teamautonomie. Die Teamautonomie beschreibt das Ausmaß, in dem ein Team als Ganzes die Freiheit hat, seine Aufgaben und Vorgehensweisen zu bestimmen (Carter et al. 2018). Sie kann anhand der Koordinationsstruktur beurteilt werden (Kim et al. 2002): Für eine niedrige Autonomie steht eine sequenzielle Koordinationsstruktur, die sich auf ein schrittweises Vorgehen bezieht, das keine Abweichung von einer system-definierten, linearen Interaktionsprozedur zulässt. Eine hohe Autonomie besteht bei uneingeschränkten (auch als parallel benannten) Koordinationsstrukturen, die es Teammitgliedern erlauben, zwischen Aufgaben hin- und herzuwechseln. Die Kooperation verfolgt in großen Teilen eine sequenzielle Arbeitsweise (siehe Abschn. 4.1). Zudem sind für die kooperationsspezifischen Aufgaben normalerweise die Interaktionsprozeduren einzuhalten, die vom Holzbauunternehmen in seiner Rolle als Totalunternehmen definiert werden. Es liegen keine Aussagen vor, inwiefern eine Veränderung der Teamautonomie durch das digitale Tool gewünscht ist.

Feedback. Feedback bezieht sich auf Informationen, die dem Team zur Leistungssteigerung dienen sollen, und können von der Aufgabe oder von Personen (z. B. Teamleiterinnen und -leitern) stammen (Carter et al. 2018; Geister et al. 2006). Unterschieden werden kann zwischen Ergebnisfeedback, das Informationen zur Aufgabenperformanz beinhaltet, und Prozessfeedback, das sich auf den Prozess der Aufgabenumsetzung bezieht (Earley et al. 1990). Sowohl zum Ergebnis als auch zum Prozess wird vorrangig fehler- oder problemorientiert Feedback gegeben. Wenn Probleme auftauchen, werden diese adressiert. Treten keine Unstimmigkeiten auf, erfolgt nur selten ein konkretes Feedback. Ab und zu bespricht das Holzbauunternehmen nach Abschluss eines Auftrags das Ergebnis und den Prozess mit langjährigen Kooperationsbeteiligten im Rahmen eines Abschlussmeetings. Durch das digitale Tool soll vor allem bei Problemen systembedingtes Feedback bereitgestellt werden, um somit schneller als bisher Feedback geben zu können.

Soziale Unterstützung. Die Dimension soziale Unterstützung gibt Auskunft, inwiefern während der Arbeit Gelegenheiten zur Verfügung stehen, andere um Rat oder Unterstützung zu bitten (Morgeson und Humphrey 2006). Die Kooperation besteht aus einem vornehmlich einfachen Netzwerk, bei dem das Holzbauunternehmen in seiner Funktion als Totalunternehmen im Zentrum der Kommunikation und 
Koordination steht. Die Aufgabenabsprachen und Möglichkeiten zur Unterstützung laufen demnach meistens bidirektional zwischen dem Holzbauunternehmen und einzelnen Subunternehmen. Sollten sich die Subunternehmen untereinander unterstützen, dann geschieht dies normalerweise außerhalb der gegebenen Kooperationsstrukturen. Es wurden keine Äußerungen zur Veränderung der sozialen Unterstützung durch das digitale Tool getätigt.

\section{Ein digitales Tool zur Unterstützung der Gebäudeplanung}

Das Holzbauunternehmen möchte zur Optimierung der Kooperation ein an BIM orientiertes digitales Tool nutzen. Es soll (1) relevante Informationen in ein 3D-visualisiertes Modell integrieren, (2) neben einer Schnittstelle zu gängigen 3D-Anwendungen durch standardisierte Eingabemasken mit Rückgriff auf Meta-Daten die Weitergabe vollständiger Informationen erleichtern und (3) über Markierungen, Text-, Bild- und/oder Sprachnachrichten einen gemeinsamen Kommunikationskanal bieten, um die Abstimmung und Dokumentation zu erleichtern. Da keine gewerkinterne 3D-Modellierung nötig sein wird, soll insbesondere computerisierten Unternehmen, die bislang keine oder kaum Berührungspunkte mit 3D-Modellierung haben und dadurch keine ausreichenden Kompetenzen in dem Bereich aufbauen konnten, eine Mitarbeit ermöglicht werden. Bei dem digitalen Tool wird es sich um eine Web-App handeln, auf die unabhängig von verschiedenen Betriebssystemen und Endgeräten zugegriffen werden kann. Insgesamt soll durch die Web-App die Zusammenführung der Planungen der unterschiedlichen Gewerke vereinfacht und weniger fehleran- fällig werden. Indem mehr Arbeit in die Planung investiert wird, sollen Probleme in der Ausführung des Gebäudebaus vermieden werden. Der Fokus liegt laut dem Totalunternehmen auf der Behebung von bestehenden Problemen. Zur grundlegenden Kooperationsart wurden keine Änderungswünsche geäußert. Das digitale Tool soll demnach die aktuelle Kooperation optimieren, ohne grundlegende Formveränderungen auszulösen.

Von den Kooperationsbeteiligten sowie Vertreterinnen und Vertretern des beteiligten Softwareunternehmens und Weiterbildungsinstituts aus dem Handwerk wurden 14 Vorund sieben Nachteile genannt, die zuerst anhand der Mensch-Technik-Organisation-Unterteilung (Ulich 2013) kategorisiert wurden. Anschließend wurde in Anlehnung an die SWOT-Analyse (strengths, weaknesses, opportunities, threats; z. B. Wollny und Paul 2015) beurteilt, welchen Einfluss die genannten Vor- und Nachteile des digitalen Tools in Kombination mit den in Abschn. 4 und 5 herausgearbeiteten Stärken und Schwächen der Kooperation auf das Ziel haben könnte, die Kooperation durch eine vereinfachte und weniger fehleranfällige Integration der verschiedenen Gewerkplanungen mittels des digitalen Tools zu verbessern (siehe Abb. 3).

Es zeigt sich (siehe Abb. 3), dass jeweils vier der insgesamt 14 genannten Vorteile vor allem in der Schnittmenge zwischen Technik und Kooperationsbeteiligten sowie Technik und Kooperationsorganisation und jeweils drei Vorteile vorrangig im Bereich der Kooperationsorganisation sowie in der Schnittmenge zwischen Kooperationsorganisation und Kooperationsbeteiligten gesehen werden. 13 der 14 genannten Vorteile des digitalen Tools betreffen Aspekte der Kooperation, die häufig problembehaftet sind. Hier gilt es entsprechend der Handlungsempfehlungen von Wollny

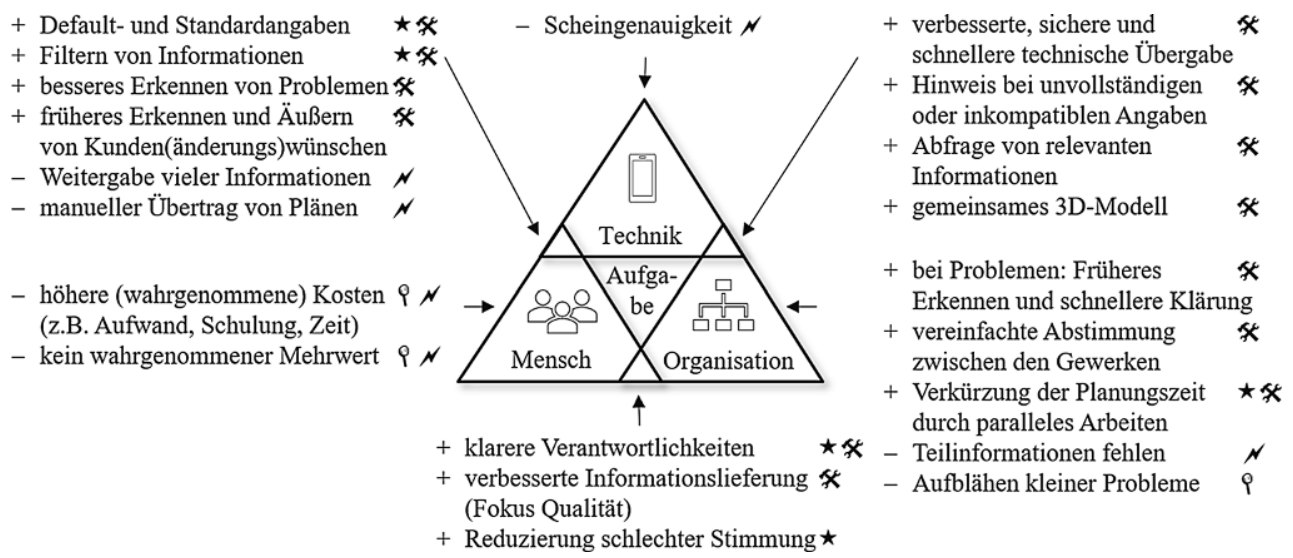

Abb. 3 Kategorisierung der antizipierten Vor- und Nachteile des digitalen Tools nach Mensch, Technik und Organisation (Ulich 2013). + Vorteil des digitalen Tools, - Nachteil des digitalen Tools, $\star$ Vorteil des digitalen Tools könnte in Kombination mit den Stärken der gewerkübergreifenden

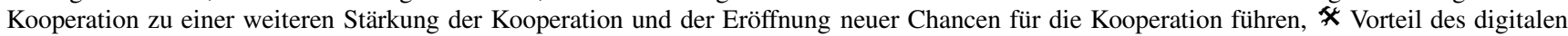
Tools könnte in Kombination mit bestehenden Problemen in der Kooperation zu einer Verbesserung bestehender Probleme oder Neutralisierung des Vorteils führen, 9 Nachteil des digitalen Tools könnte in Kombination mit den Stärken der gewerkübergreifenden Kooperation zu einer Neutralisierung des Nachteils oder zur Schwächung der Stärken der Kooperation führen, $\mathbb{N}$ Nachteil des digitalen Tools könnte in Kombination mit bestehenden Problemen in der Kooperation zu einer Verschlimmerung bestehender oder zur Entstehung neuer Probleme führen 
und Paul (2015) zu überlegen, wie die Vorteile des digitalen Tools genutzt werden können, um die Probleme der Kooperation zu beseitigen. Beispielsweise soll das digitale Tool die oftmals als beeinträchtigt beschriebene Informationslieferung zwischen den Gewerken verbessern. Die Eingabemasken könnten hierbei strukturierend wirken. Eventuell ist jedoch zudem eine Schulung notwendig, um den Vorteil zu realisieren. Fünf der 14 Vorteile beziehen sich unter anderem auch auf Bereiche der Kooperation, die normalerweise problemlos verlaufen. In Anlehnung an Wollny und Paul (2015) sollte überlegt werden, wie die Vorteile zur weiteren Stärkung der Kooperation und der Eröffnung neuer Chancen für die Kooperation dienen können. Durch das digitale Tool sollen beispielsweise klarere Verantwortlichkeiten als vorher geschaffen werden. Der hohe Grad an Strukturierung kann als Stärke der Kooperation betrachtet werden, die durch die digitale Toolnutzung noch weiter optimiert werden kann und beim Identifizieren von Verantwortlichkeiten durch das digitale Tool unterstützen kann.

Es wurden zudem sieben mögliche Nachteile des digitalen Tools genannt (siehe Abb. 3), von denen sich jeweils zwei vorrangig auf die Kooperationsbeteiligten, die Kooperationsorganisation sowie die Schnittmenge zwischen Kooperationsbeteiligten und Technik beziehen. Ein Nachteil wurde spezifisch für die Technik identifiziert. Sechs der sieben Nachteile treffen auf häufig problembehaftete Bereiche der Kooperation, bei denen in Anlehnung an Wollny und Paul (2015) Maßnahmen ergriffen werden sollten, die die Probleme der Kooperation ausgleichen, um den Nachteil des digitalen Tools erfolgreich entgegentreten zu können. Alternativ kann überlegt werden, wie das digitale Tool, welches im Fallbeispiel erst als Konzeption vorliegt, abgeändert werden kann, um diesen Nachteil zu reduzieren, zu eliminieren oder durch andere Vorteile zu kompensieren.
Beispielsweise könnte es zum manuellen Übertrag von anderweitig erstellten 2D-Modellen in das digitale Tool kommen. Es ist nun zu überlegen, ob es zum Beispiel möglich ist, die Planungen direkt im digitalen Tool vorzunehmen oder Schnittstellen zu anderen 2D und 3D-Modellierungsprogrammen zu integrieren. Drei der sieben Nachteile treffen zudem auch auf Stärken der Kooperation, die in Anlehnung an Wollny und Paul (2015) zum Abpuffern der Nachteile genutzt werden können. Beispielsweise könnte die Stärke der Kooperation, durch die Zusammenarbeit gemeinsam der Kundschaft ein Produkt ,aus einer Hand“ bieten zu können, den Nachteil abfedern, dass einige Kooperationsbeteiligte eventuell für ihr eigenes Unternehmen oder ihr eigenes Gewerbe keinen oder wenig Mehrwert in der digitalen Toolnutzung sehen.

Insgesamt wurden hauptsächlich gewerkunspezifische Vor- und Nachteile genannt und der Fokus auf die Konsequenzen für interne Stakeholder gelegt. Mit Blick auf das Technologieakzeptanzmodell (TAM, engl. Technology Acceptance Model, z. B. Davis und Venkatesh 1996; Venkatesh und Bala 2008) wurde hauptsächlich die Nützlichkeit und weniger die Benutzerfreundlichkeit (nur beim Vorteil der einfacheren Bedienung durch Default- und Standardangaben und bei den beiden Nachteilen der verpflichtenden Eingabe vieler Informationen und dem manuellen Übertrag bestehender Pläne) des digitalen Tools betrachtet. Im Folgenden wird aufgezeigt, welchen Einfluss das digitale Tool auf den Arbeitsprozess haben kann.

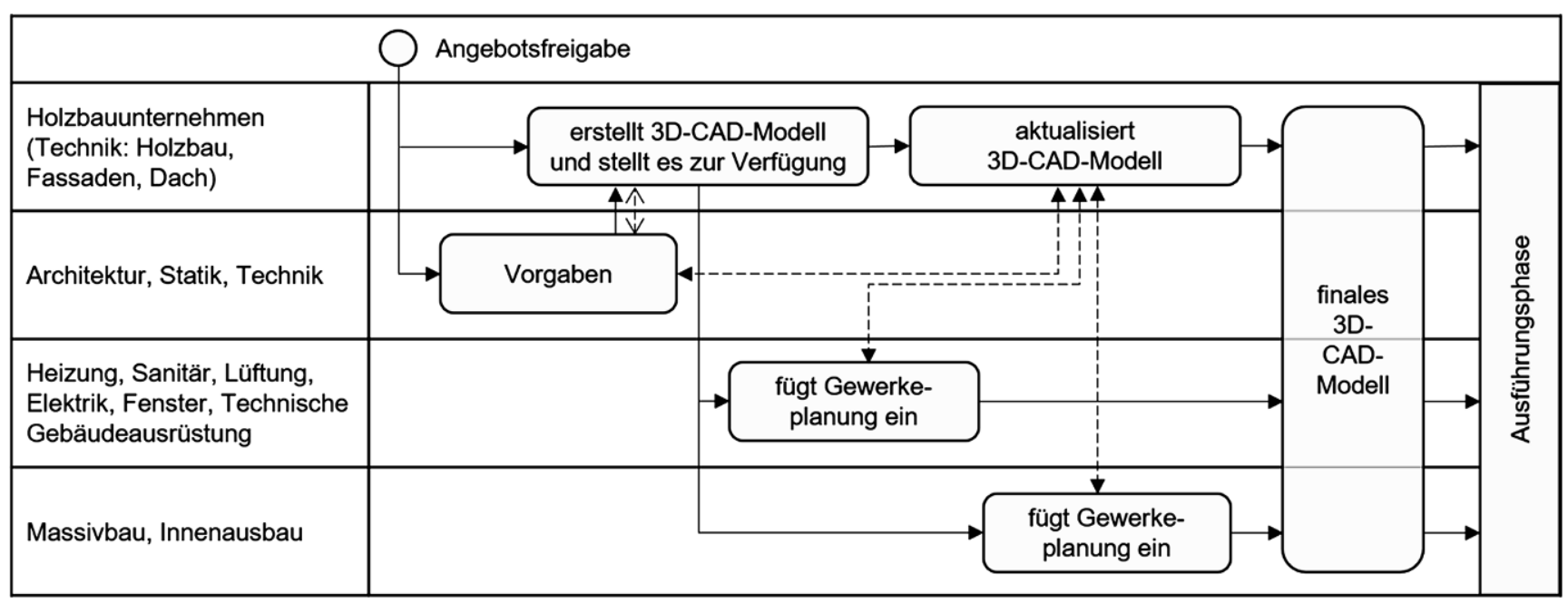

Abb. 4 Möglicher Arbeitsprozess nach Einführung des digitalen Tools in die gewerkübergreifende Kooperation bei der Gebäudeplanung. Die durchgehenden Pfeile stellen die standardisierte Informationslieferkette dar. Die gestrichelten beidseitigen Pfeile symbolisieren bedarfsbedingte Nachfrageschleifen 


\section{Mögliche Veränderung des Kooperations- prozesses durch das digitale Tool}

Durch die Einführung des digitalen Tools sollte sich der Kooperationsprozess verändern. Basierend auf den Workshopangaben könnte der veränderte Arbeitsprozess folgendermaßen aussehen (siehe Abb. 4): Im ersten Schritt wird weiterhin - wie in Abschn. 4.1 beschrieben und in Abb. 1 dargestellt - vom Holzbauunternehmen, in Abstimmung mit den Architektinnen und Architekten sowie Statikerinnen und Statiker, ein detaillierter Plan erstellt. Das daraus entstandene 3D-CAD-Modell wird dann allerdings im Unterschied zum bisherigen Prozess den Kooperationsbeteiligten über das digitale Tool zur Verfügung gestellt. Die Kooperationsbeteiligten können nun selbst ihre Gewerkplanungen über die strukturierten Eingabemasken in das 3D-CAD-Modell einfügen. Der Fortschritt ist von allen Beteiligten einsehbar, sodass schneller und direkter bei Fragen und Absprachen kommuniziert werden kann. Allerdings soll die Kommunikation weiterhin beim Holzbauunternehmen zusammenlaufen. Das finale 3D-CAD-Modell steht anschlieBend allen an der Umsetzung des Bauplanes beteiligten Kooperationsbeteiligten zur Verfügung.

Im Folgenden wird durch Rückgriff auf wissenschaftliche Konzepte und Befunde betrachtet, inwiefern sich die Arbeitsgestaltung in der Kooperation durch das digitale Tool verändern kann.

\section{Mögliche Veränderung der Arbeitsgestaltung innerhalb der Kooperation durch das digitale Tool}

Es wurde ein Abgleich der möglichen Veränderungen der Arbeitsgestaltung mit den gewünschten Änderungen vorgenommen (siehe Tab. 1).

\subsection{Interdependenz}

Aufgabeninterdependenz. Das digitale Tool sollte durch die strukturierte Abfrage relevanter Informationen, der direkten Integration und Visualisierung von Informationen und der automatischen, systemgesteuerten Benachrichtigung bei Veränderungen wie gewünscht die Koordination in der Planungsphase der Zusammenarbeit unterstützten und somit erleichtern können. Dadurch kann der bestehenden Aufgabeninterdependenz besser entsprochen werden. Es ist zudem zu erwarten, dass die Aufgabeninterdependenz stärker sichtbar wird, da beispielsweise über die 3DVisualisierung leichter dargestellt werden kann, welche Informationen aus einem Gewerk für ein anderes relevant sind und wie der Arbeitsprozess ins Stocken geraten kann, wenn die einzelnen Gewerkplanungen an den Schnittstellen zu den anderen inkompatibel sind.

Zudem kann BIM durch die Möglichkeit zu schnelleren und direkteren Absprachen sowie stärker parallelem Arbeiten (siehe Fischer et al. 2017) zu einer Erhöhung der Aufgabeninterdependenz führen, die sich wiederum positiv auf die Teammotivation auswirken kann (Hertel et al. 2005, 2004). Diese mögliche Veränderung ist vom Holzbauunternehmen nicht explizit vorgesehen, sollte jedoch durchdacht werden, da sie beispielsweise mit einem erhöhten Koordinationsaufwand einhergeht. Zudem können viele reziproke Arbeitsprozesse zu einer Verringerung der Teameffektivität führen, wenn die Teammitglieder sich nicht face-to-face austauschen können (Hertel et al. 2005).

Ergebnisinterdependenz. Alle Eingaben in das digitale Tool werden getrennt nach Kooperationsbeteiligten gespeichert und zur Einsicht zur Verfügung gestellt. Dadurch sollte ein effizienteres Aufdecken der Fehlerquelle bei Problemen ermöglicht werden. Da die finanzielle Entlohnung der Unternehmen an ihre Einzelleistungen gekoppelt ist, sollte das Klima in der Kooperation beachtet werden. Bei niedrigem Vertrauen unter den Beteiligten könnte diese höhere Sichtbarkeit und Nachverfolgbarkeit der Einzelleistungen dazu führen, dass die Kooperationsbeteiligten das digitale Tool als Methode zum Finden von Schuldigen bei Problemen wahrnehmen und deshalb ungern Informationen über das digitale Tool preisgeben.

Wenn auch nicht intendiert, könnte durch die Arbeit an einem gemeinsamen Gebäudemodell die Wahrnehmung und Kommunikation des Endergebnisses als Gruppenergebnis erhöht werden. Dieser Effekt kann noch verstärkt werden, wenn das digitale Tool zu Projektabschluss zur gemeinsamen Reflexion genutzt werden würde. Diese erhöhte Ergebnisinterdependenz kann sich vor allem wenn die Beiträge, die durch das digitale Tool vermutlich verstärkt einzeln identifizierbar sind, unterstützend auf die Teamarbeit auswirken (Kahai et al. 2003). Allerdings sollte hier darauf geachtet werden, dass kein Bruch zwischen dem Eindruck, der durch das digitale Tool vermittelt wird, und der vertraglichen Regelung, die eine Belohnung der Einzelleistungen vorsieht, entsteht.

\subsection{Wissensmerkmale}

Aufgabenunsicherheit. Änderungen am Bauplan können durch das digitale Tool direkt allen angezeigt werden. Zudem sorgt die Benachrichtigungsfunktion des digitalen Tools für eine schnellere Information aller Kooperationsbeteiligten bei unvorhersehbaren Ereignissen und kann Abstimmungsprozesse auslösen. Dadurch kann eine effizientere und effektivere Reaktion erreicht werden. Allerdings kann durch das Hinzufügen des digitalen Tools zur Aufga- 
benumgebung die Aufgabe anfangs unberechenbarer (z. B. was passiert, wenn wer was macht) und insgesamt dynamischer (z.B. gleichzeitiger Zugriff, schnellerer Ablauf) werden. Gegebenenfalls können (Face-to-face-) Meetings (Painter et al. 2016), das Aufbauen von Routinen sowie das Bereitstellen von Dokumentationen unterstützend wirken.

Aufgabenroutine. Nicht nur ist das digitale Tool zu Beginn der Nutzung unbekannt, auch BIM ist für viele der Kooperationsbeteiligten noch neuartig. Insbesondere wenn viel digital vermittelt kommuniziert wird, kann eine hohe Neuartigkeit der Aufgabe mit geringem Vertrauen in die Teammitglieder und niedrigem Aufgabenbewusstsein einhergehen (Espinosa et al. 2007). Damit dieses Phänomen nicht eintritt oder schnell überwunden werden kann, sollten zeitnah Routinen aufgebaut sowie Dokumentationen und Anleitungen zum Arbeitsprozess zur Verfügung gestellt werden. Gegebenenfalls kann anfangs die Virtualität durch mehr Face-to-face-Meetings verringert oder ihr durch die Nutzung adäquater IKT besser entsprochen werden. Je stärker die Routiniertheit ist, desto besser verläuft die Kommunikation im Team (Handke et al. 2020).

Aufgabenkomplexität. Mit dem zu entwickelnden digitalen Tool kann den Anforderungen der vorliegenden Aufgabenkomplexität der Kooperation besser entsprochen und vor allem die Mehrdeutigkeit reduziert werden, da durch das Einfordern und Zusammentragen der Informationen an einem digitalen Ort, der für alle Beteiligten einsehbar ist, schneller sichtbar wird, ob Passung gegeben ist und wo Informationen fehlend oder unzureichend sind. Allerdings ist davon auszugehen, dass sich durch den zusätzlichen Aufwand und das Finden neuer Arbeitsprozesse im Umgang mit dem digitalen Tool die Aufgabenkomplexität anfangs erhöhen wird. Die Subunternehmen stehen aktuell vorrangig mit dem Generalunternehmen im Austausch und werden voraussichtlich durch das digitale Tool stärker auf weitere Parteien achten müssen. Eine höhere Aufgabenkomplexität geht mit höheren Anforderungen an die Kommunikation (Bosch-Sijtsema et al. 2011; Maznevski und Chudoba 2000) und Aufmerksamkeit einher und verlangt vom Team mehr Zeit zur Reflexion der Arbeitsschritte (Bosch-Sijtsema et al. 2011). Vor allem in der anfänglichen Nutzung sollte also ein erhöhter Kommunikations-, Aufmerksamkeits- und Reflexionsbedarf antizipiert werden.

Problemlösung. Vor allem bei intellektuellen Aufgaben kann eine stark über digitale Tools vermittelte Kommunikation zu Problemen führen, wenn sie nicht reichhaltig genug sind und keine synchrone Kommunikation erlauben, um den benötigten Diskussions- und Annäherungsprozess zu tragen (Handke et al. 2020; Laughlin und Ellis 1986). Das geplante digitale Tool birgt hier Risiken und Chancen.
Zum einen kann der Problemlöseprozess durch eine angemessenere Reichhaltigkeit (z. B. 3D-Modell) und schnellere Kommunikation (z.B. automatische Benachrichtigungen) besser als vorher unterstützt werden. Zudem verstärkt die höhere Virtualität, die durch die Nutzung des digitalen Tools ausgelöst werden kann, den Fokus auf die Aufgabe (Huang und Wei 2000), wodurch effizientere Lösungen ermöglicht werden. Zum anderen kann durch die bestehende Virtualität der benötigte Raum zur Diskussion in der Lösungsfindung eingeschränkt sein, vor allem wenn sich die im digitalen Tool enthaltenen Kommunikationsmöglichkeiten als nicht reichhaltig genug herausstellen sollten. Bei Aufgaben, die einen besonders hohen Diskussionsund Abstimmungsbedarf besitzen, sollte daher das digitale Tool in Kombination mit face-to-face Meetings verwendet werden.

Informationsverarbeitung. Durch das digitale Tool sollen schneller eindeutigere Informationen eingefordert und abgeliefert werden können. Damit sollte dem hohen Niveau an Informationsbeschaffung besser als vorher entsprochen werden können. Dem hohen Niveau an Informationsverarbeitung wird das digitale Tool beispielsweise durch die Visualisierung gerecht.

Durch die hohe Informationsmenge, die durch das BIMorientierte Tool zur Verfügung stehen wird, könnte vor allem für die Subunternehmen das Ausmaß an benötigter Informationsverarbeitung in der Kooperation zunehmen. Bei digital vermittelter Kommunikation kann das Bieten vieler Informationen bei Aufgaben, die eine hohe Informationsverarbeitung erfordern, leistungsförderlich sein (Schreiber und Engelmann 2010), jedoch nicht, wenn sie alle gleichzeitig präsentiert werden (Ferreira et al. 2011). Das digitale Tool sollte also zur Vermeidung einer kognitiven Überlastung so gestaltet sein, dass alle relevanten Informationen einsehbar sind, jedoch flexibel ein- oder ausblendbar sind.

\subsection{Arbeitsbezogene Anforderungen}

Rollenambiguität. Das digitale Tool kann klare Verhaltensanweisungen für den gewerkübergreifenden Austausch bieten, da beispielsweise durch die Informationsabfragen in der Eingabemaske ersichtlich wird, welche Informationen von anderen Kooperationsbeteiligten benötigt werden. Allerdings könnte die Rollenklarheit anfänglich reduziert sein, da durch das digitale Tool nicht nur die Nutzung einer neuen Technologie hinzukommt, sondern zudem BIM-spezifische Prozess- und Systemänderungen auftreten können. Neben kleineren Unklarheiten, zum Beispiel welche Verantwortlichkeiten durch das digitale Tool für den einzelnen Beteiligten entstehen, können sich neue Rollen, wie beispielsweise die der BIM-Koordination (siehe Jacobsson und Merschbrock 2018) herausbilden, deren Ausgestaltung 
und Verankerung im Prozess und System erst ausgehandelt werden müssen. Deshalb scheint es notwendig zu sein, sich zumindest anfangs explizit über die Rollenzuteilung (z.B. wer übernimmt wann welche Aufgabe) auszutauschen, um aufgabenbezogene Transparenz und Verantwortlichkeit zu schaffen.

Insgesamt kann das digitale Tool auf längere Sicht unterstützen, akkurate mentale Repräsentationen der Arbeitsprozesse zu entwickeln, die sich auf das Wissen über die zeitliche Planung des Projekts, die individuellen und gemeinsamen Ziele, die Aufgaben, die Handlungsstrategien, die Rollen der einzelnen Teammitglieder und die jeweils aktuellen Anforderungen im Projekt, die idealerweise zudem zwischen allen Kooperationsbeteiligten verbindlich abgestimmt sein sollten, beziehen (siehe mentale Modelle, Antoni und Ellwart 2017). Ebenfalls kann das digitale Tool durch die Darstellung der Informationen aller Gewerke dabei helfen, die sogenannten T-förmigen Kompetenzprofile (Barile et al. 2012; Demirkan und Spohrer 2015; Hansen und von Oetinger 2001) weiterzuentwickeln, indem neben spezialisierten Fachkompetenzen auf einer vertikalen Ebene, auf einer horizontalen Ebene Kompetenzen aus anderen Gewerken aufgebaut werden. Die Sanitärplanung muss beispielsweise wissen, welche Informationen aus dem eigenen Gewerk für den Holzbau und die anderen Kooperationsbeteiligten relevant sind und welche Informationen sie selbst aus anderen Gewerken einfordern muss.

Zeitdruck. Zeitdruck wird häufig als Stressquelle wahrgenommen, die zwar zu mehr Engagement der Teammitglieder führen kann, jedoch vor allem bei Teams, die verstärkt über digitale Tools kommunizieren, schädlich auf den Entscheidungsprozess und das Endergebnis wirken kann (Argote et al. 1989; Chu und Spires 2001). Durch das digitale Tool stehen Möglichkeiten zur Verfügung, sich auch unter Zeitdruck effizient zu koordinieren und abzustimmen sowie die Dokumentation der Ereignisse aufrechtzuerhalten, da Veränderungen am 3D-Modell automatisch gespeichert werden, zu systemgesteuerten Benachrichtigungen der relevanten Kooperationsbeteiligten führen und nachträglich über ein Verlaufslogbuch einsehbar sind. Allerdings muss das digitale Tool auch unter erhöhtem Zeitdruck und in Problemsituationen konsequent genutzt werden, um diese Effekte haben zu können. In der Literatur wurde bereits darauf hingewiesen, dass BIM nur dann zu einer Verbesserung des Bauprozesses führen kann, wenn die Informationen im digitalen Tool durchgängig aktuell gehalten werden (Chen et al. 2015). Deshalb sollten proaktiv Anweisungen erarbeitet werden, wie das digitale Tool unter Zeitdruck und in Problemsituationen verwendet werden soll. Zudem bietet das digitale Tool hauptsächlich den Anlass und einen Kanal zur Kommunikation, jedoch keine Struktur. Deshalb reicht der Einsatz des digitalen Tools nicht aus. Es muss zu- dem erarbeitet werden, wie insgesamt das Vorgehen unter Zeitdruck und bei Problemsituationen sein soll, damit der Entscheidungsprozess und das Ergebnis nicht beeinträchtigt werden.

\subsection{Arbeitsbezogene Ressourcen}

Teamautonomie. Häufig führt die Erhöhung der Teamautonomie zu einer Verbesserung des Funktionierens eines Teams (Handke et al. 2020), wie beispielsweise zu einer höheren Kommunikationseffektivität (Kim et al. 2002) und zur Entwicklung von Vertrauen im Team (Breuer et al. 2020). Das digitale Tool kann die Teamautonomie erhöhen. Es könnte zu Abweichungen von der momentanen Arbeitsweise kommen, indem mehr reziproke und parallele Prozesse ermöglicht werden. Zum Beispiel könnte es niederschwelliger zu Abstimmungsprozessen zwischen den Kooperationsbeteiligten bei (erwartbaren) Problemen kommen, da über das für alle verfügbare 3D-Modell schneller und einfacher Diskussionsbedarf auffällt. Zudem werden die Kooperationsbeteiligten voraussichtlich gleichzeitig für einen längeren Zeitraum Zugriff auf das digitale Tool haben, sodass an einigen Stellen paralleles Arbeiten ermöglicht wird.

Feedback. Prozess- und Ergebnisfeedback wirken sich meistens positiv auf das Team aus (Handke et al. 2020), da Feedback beispielsweise mit einem verbesserten Umgang mit Konflikten einhergeht (Martínez-Moreno et al. 2015). Durch das digitale Tool steht schnell systembedingtes Feedback zur Verfügung. Über die Eingabemasken im digitalen Tool wird leicht erkennbar sein, ob vollständige Informationen vorliegen oder Angaben in bestimmten Bereichen fehlen. Zudem wird grafisch markiert, falls es zu harten (z.B. Wand und Treppe nehmen den gleichen Raum ein) oder weichen (z. B. die Tür lässt sich nicht öffnen, weil die Treppe im Öffnungsradius steht) Kollisionen kommt. Dieses Feedback steht auch problemunabhängig zur Verfügung, erhöht die Häufigkeit an Rückmeldungen zur eigenen Leistung und kann Grundlage für Feedback sein, das sich die Kooperationsbeteiligten während des Prozesses oder beim Abschlussmeeting geben.

Soziale Unterstützung. Soziale Unterstützung hat viele Vorteile für Teams und ihre Arbeitsleistung (Handke et al. 2020; Parker und Grote 2020). Das digitale Tool kann in zweierlei Hinsicht die soziale Unterstützung in der Kooperation verstärken. Durch die gemeinsame Arbeit an dem 3D-Modell kann zum einen das Wir-Gefühl und die „Presence Awareness“ (das Gefühl, dass Personen physisch anwesend und füreinander ansprechbar sind, als ob sie kopräsent wären; Malhotra und Majchrzak 2014) gestärkt und somit die Sicht auf die anderen Kooperationsbeteiligten als mögliche Quellen sozialer Unterstützung verstärkt werden. 
Vor allem bei interdisziplinären Teams können IKT, die das Bewusstsein für die Anwesenheit anderer (,Presence Awareness“) erhöhen, die Teamleistung steigern (Malhotra und Majchrzak 2014). Zum anderen wird ersichtlich, wer für welche Themen zuständig ist, sodass ein komplexeres Netzwerk entstehen kann, das mehr Möglichkeiten der bi- oder multilateralen Unterstützung zwischen allen Kooperationsbeteiligten bietet.

\section{Fazit}

Der vorliegende Beitrag dient der Darstellung, wie der Kooperationsprozess und die Arbeitsgestaltung systematisch auf mögliche Veränderungen eines digitalen Tools hin untersucht werden können. Dem soziotechnischen Systemansatz der gemeinsamen Optimierung (joint optimization) von Technologie und Arbeitsgestaltung (Davis 2019; Davis et al. 2014; Parker und Grote 2020; Ulich 2013) folgend wurde bezüglich der gewerk- und unternehmensübergreifenden Kooperation im Baugewerbe ein deskriptiver Blick auf den Ist-Zustand, ein normativer Blick auf den gewünschten Zustand und ein prädiktiver Blick auf den möglichen Zustand nach der Einführung des digitalen Tools (Baxter und Sommerville 2011) eingenommen.

Die Analyse zeigt auf, dass die meisten gewünschten Änderungen, wie eine verbesserte Koordination oder ein optimierter Umgang mit der bestehenden Aufgabenkomplexität, durch das digitale Tool zwar ermöglicht werden können, jedoch vielfältige weitere Arbeitsgestaltungsänderungen ausgelöst werden können. So können sowohl weitere positive Veränderungen wie der Aufbau adäquater mentaler Modelle durch das gemeinsame interdisziplinäre Arbeiten am 3D-Modell als auch negative Konsequenzen wie die (anfänglich) höhere Aufgabenkomplexität durch die zu erwar- tende stärkere Interaktivität zwischen den Subunternehmen resultieren. Auch können Abweichungen zum gewünschten Arbeitsprozess eintreten, indem sich durch die Möglichkeit, parallel an dem 3D-Modell zu arbeiten, der Arbeitsprozess stärker als angenommen von einer sequenziellen zu einer reziproken Arbeitsweise wandelt.

Diese frühzeitige Analyse in der Startphase des Digitalisierungsprojektes kann dabei unterstützen, auch in hektischen Phasen der Implementierung neuer Technologien handlungsfähig zu sein, da rechtzeitig Strategien zum Umgang mit den Konsequenzen erarbeitet werden können (siehe hierzu z. B. Chien et al. 2014). In Übereinstimmung mit vorheriger Forschung (z. B. Forgues et al. 2016; Parker und Grote 2020) wird in diesem Fallbeispiel deutlich, dass neben der Einführung des digitalen Tools aktiv weitere Maßnahmen ergriffen werden sollten, damit das digitale Tool sein Potenzial zur Verbesserung der Kooperation entfalten kann. Die vorliegende Analyse bietet Ansatzpunkte, z. B. mit dem Fokus auf die Arbeitsgestaltungsdimensionen, in welchen Bereichen Maßnahmen sinnvoll sind. Beispielsweise sollten Dokumentationen erarbeitet und bereitgestellt werden, um Routinen auf- und Unsicherheit abzubauen. Bei komplexen interdisziplinären Aufgaben sollte insbesondere in der Anfangszeit gemeinsam in Face-to-face Meetings am digitalen Tool gearbeitet werden, um dem hohen Abstimmungs- und Diskussionsbedarf gerecht zu werden. Auch scheint ein aktiver Austausch über die neuen Rollenzuteilungen vorteilhaft, um aufgabenbezogene Transparenz und Verantwortlichkeit zu schaffen.

Diese frühzeitige Analyse der Konsequenzen digitaler Tools könnte in Kombination mit partizipativen Maßnahmen zur Erhöhung der Technologieakzeptanz (siehe z.B. Venkatesh und Bala 2008) eine präzise Prädiktion, die Erarbeitung von realistischen Eingriffsmöglichkeiten und eine Stärkung des Commitments der Stakeholder ermöglichen.
Abb. 5 Zusammenfassung der genutzten Konzepte zur Abschätzung der Digitalisierungskonsequenzen der Arbeit für das Individuum und das Kooperationsteam

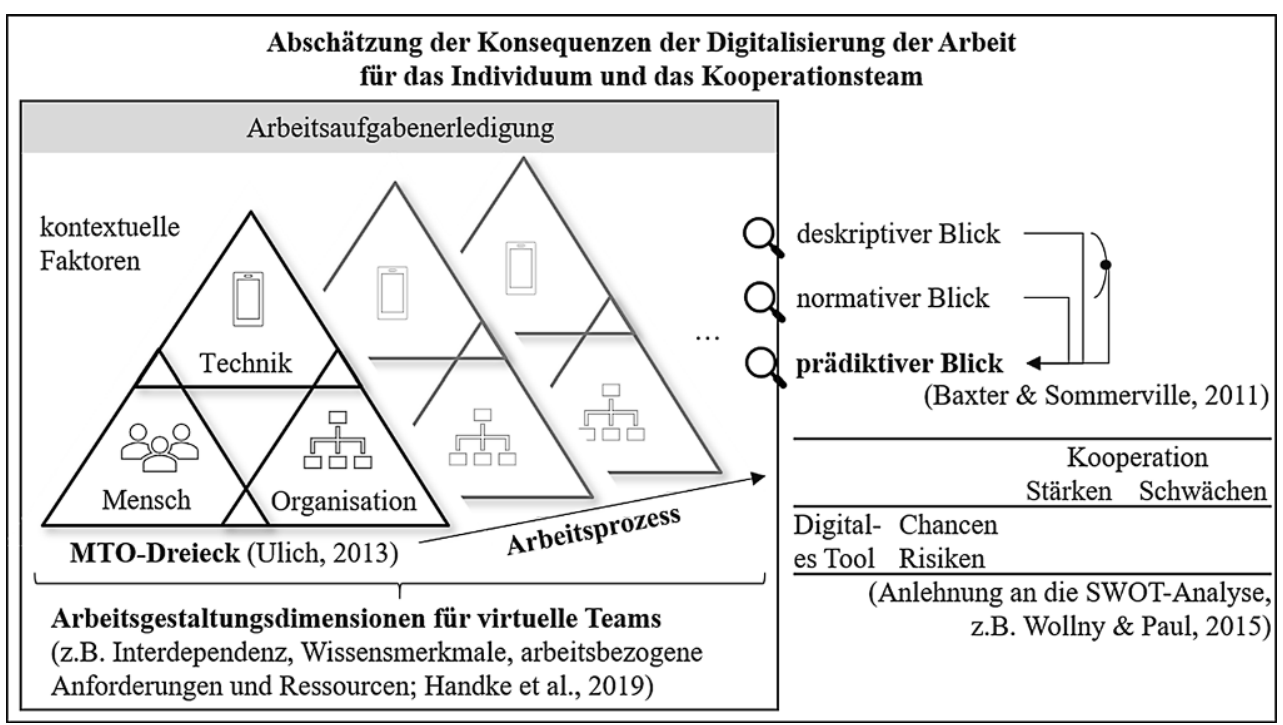


Die Ergebnisse der Analyse können zudem eine Entscheidungsgrundlage zur Ableitung konkreter Maßnahmen bieten, die in Abgleich mit dem Technologieakzeptanzmodell (z.B. Davis und Venkatesh 1996) positiv auf zentrale Einflussgrößen der Technologieakzeptanz - wie die wahrgenommene Nützlichkeit und Benutzerfreundlichkeit - wirken sollten.

Die Auseinandersetzung mit den möglichen Konsequenzen der Digitalisierung der Arbeit ist ein wichtiger Schritt in der Startphase von Digitalisierungsprojekten (Parker und Grote 2020; Schlicher et al. 2018; Venkatesh und Bala 2008). Anhand des Fallbeispiels kann aufgezeigt werden, wie theoretische Konzeptionen zur Beurteilung der Digitalisierungskonsequenzen gewinnbringend in die Praxis eingebracht werden können. Darauf aufbauend kann die theoretische Ausdifferenzierung dieses Schrittes vorangetrieben werden. Die hier verwendeten Konzepte (siehe Abb. 5) bieten ein umfangreiches Raster zur Beleuchtung der Konsequenzen mit Fokus auf den Arbeitsprozess, die Arbeitsgestaltung, die Menschen, die Organisation und die Technik. In diesen Bereichen wird der deskriptive und gewünschte normativen Zustand anvisiert, um prädiktive Annahmen über positive und negative Veränderungen der Kooperation durch das digitale Tool ableiten zu können. Längsschnittliche Forschung ist jedoch vonnöten, um die Bedeutung dieser frühzeitigen Auseinandersetzung mit den Konsequenzen der Implementierung eines digitalen Tools näher zu bestimmen.

Förderung Das Vorhaben (IN-DIG-O, 02L17C590) wird im Rahmen des Programms „Zukunft der Arbeit“ unter dem Dachprogramm „Innovationen für die Produktion, Dienstleistung und Arbeit von morgen“ vom Bundesministerium für Bildung und Forschung und dem Europäischen Sozialfonds gefördert.

\section{Funding Open Access funding provided by Projekt DEAL.}

Open Access Dieser Artikel wird unter der Creative Commons Namensnennung 4.0 International Lizenz veröffentlicht, welche die Nutzung, Vervielfältigung, Bearbeitung, Verbreitung und Wiedergabe in jeglichem Medium und Format erlaubt, sofern Sie den/die ursprünglichen Autor(en) und die Quelle ordnungsgemäß nennen, einen Link zur Creative Commons Lizenz beifügen und angeben, ob Änderungen vorgenommen wurden.

Die in diesem Artikel enthaltenen Bilder und sonstiges Drittmaterial unterliegen ebenfalls der genannten Creative Commons Lizenz, sofern sich aus der Abbildungslegende nichts anderes ergibt. Sofern das betreffende Material nicht unter der genannten Creative Commons Lizenz steht und die betreffende Handlung nicht nach gesetzlichen Vorschriften erlaubt ist, ist für die oben aufgeführten Weiterverwendungen des Materials die Einwilligung des jeweiligen Rechteinhabers einzuholen.

Weitere Details zur Lizenz entnehmen Sie bitte der Lizenzinformation auf http://creativecommons.org/licenses/by/4.0/deed.de.

\section{Literatur}

Aagesen, G., \& Krogstie, J. (2015). BPMN 2.0 for modeling business processes. In J. vom Brocke \& M. Rosemann (Hrsg.), Handbook on business process management (Bd. 1, S. 219-250). Berlin, Heidelberg, New York: Springer. https://doi.org/10.1007/978-3-64245100-3_10.

Antoni, C.H., \& Ellwart, T. (2017). Informationsüberlastung bei digitaler Zusammenarbeit - Ursachen, Folgen und Interventionsmöglichkeiten. Gruppe. Interaktion. Organisation. Zeitschrift für Angewandte Organisationspsychologie (GIO), 48(4), 305-315. https://doi.org/10.1007/s11612-017-0392-4.

Argote, L., Turner, M.E., \& Fichman, M. (1989). To centralize or not to centralize: The effects of uncertainty and threat on group structure and performance. Organizational Behavior and Human Decision Processes, 43, 58-74. https://doi.org/10.1016/07495978(89)90058-7.

Babič, N. C., \& Rebolj, D. (2016). Culture change in construction industry: from 2D toward BIM based construction. Journal of Information Technology in Construction (itcon), 21(6), 86-99.

Bakker, A.B., \& Demerouti, E. (2007). The job demands-resources model: state of the art. Journal of Managerial Psychology, 22, 309-328. https://doi.org/10.1108/02683940710733115.

Barile, S., Franco, G., Nota, G., \& Saviano, M. (2012). Structure and dynamics of a "T-shaped" knowledge. From individuals to cooperating communities of practice. Service Science, 4(2), 161-180. https://doi.org/10.1287/serv.1120.0014.

Baxter, G., \& Sommerville, I. (2011). Socio-technical systems: From design methods to systems engineering. Interacting with Computers, 23(1), 4-17. https://doi.org/10.1016/j.intcom.2010.07.003.

Borup, M., Brown, N., Konrad, K., \& Van Lente, H. (2006). The sociology of expectations in science and technology. Technology analysis \& strategic management, 18(3-4), 285-298. https://doi.org/ 10.1080/09537320600777002.

Bosch-Sijtsema, P. M., Fruchter, R., Vartiainen, M., \& Ruohomäki, V. (2011). A framework to analyze knowledge work in distributed teams. Group \& Organization Management, 36, 275-307. https:// doi.org/10.1177/1059601111403625.

Brandstädter, S., Schleiting, Y., \& Sonntag, K. (2018). Interdisziplinäre Kompetenz in der Wirtschaft. Zeitschrift für Arbeitswissenschaft, 72(1), 35-43. https://doi.org/10.1007/s41449-017-0080-9.

Breuer, C., Hüffmeier, J., Hibben, F., \& Hertel, G. (2020). Trust in teams: a taxonomy of perceived trustworthiness factors and risktaking behaviors in face-to-face and virtual teams. Human Relations, 73(1), 3-34. https://doi.org/10.1177/0018726718818721.

Bryde, D., Broquetas, M., \& Volm, J. M. (2013). The project benefits of building information modelling (BIM). International journal of project management, 31, 971-980.

Bråthen, K., \& Moum, A. (2016). Bridging the gap: bringing BIM to construction workers. Engineering, Construction and Architectural Management, 23(6), 751-764. https://doi.org/10.1108/ ECAM-01-2016-0008.

Carter, K. M., Mead, B. A., Stewart, G.L., Nielsen, J.D., \& Solimeo, S.L. (2018). Reviewing work team design characteristics across industries: combining metaanalysis and comprehensive synthesis. Small Group Research, 50, 138-188. https://doi.org/10.1177/ $1046496418797431 / 257090$.

Chan, A.P., Ma, X., Yi, W., Zhou, X., \& Xiong, F. (2018). Critical review of studies on building information modeling (BIM) in project management. Frontiers engineering management, 5(3), 394-406. https://doi.org/10.15302/J-FEM-2018203.

Chen, K., Lu, W., Peng, Y., Rowlinson, S., \& Huang, G. Q. (2015). Bridging BIM and building: from a literature review to an integrated conceptual framework. International journal of project management, 33(6), 1405-1416. https://doi.org/10.1016/j.ijproman. 2015.03.006. 
Chien, K.F., Wu, Z.H., \& Huang, S.C. (2014). Identifying and assessing critical risk factors for BIM projects: empirical study. Automation in Construction, 45, 1-15. https://doi.org/10.1016/j. autcon.2014.04.012.

Chu, P.C., \& Spires, E.E. (2001). Does time constraint on users negate the efficacy of decision support systems? Organizational Behavior and Human Decision Processes, 85, 226-249. https://doi.org/ 10.1006/obhd.2000.2940.

Cordery, J.L., Morrison, D., Wright, B. M., \& Wall, T.D. (2010). The impact of autonomy and task uncertainty on team performance: a longitudinal field study. Journal of Organizational Behavior, 31, 240-258. https://doi.org/10.1002/job.657.

Courtright, S.H., Thurgood, G.R., Stewart, G.L., \& Pierotti, A.J. (2015). Structural interdependence in teams: an integrative framework and meta-analysis. Journal of Applied Psychology, 100, 1825-1846. https://doi.org/10.1037/ap10000027.

Crotty, R. (2012). The impact of building information modelling: transforming construction. Abingdon: Spon.

Davies, K., McMeel, D. J., \& Wilkinson, S. (2017). Making friends with Frankenstein: hybrid practice in BIM. Engineering, construction and architectural management, 24, 78-93. https://doi. org/10.1108/ECAM-04-2015-0061.

Davis, M.C. (2019). Socio-technical systems thinking and the design of comtemporary workspace. In O. B. Ayoko \& N. M. Ashkanasy (Hrsg.), Organizational behaviour and the physical environment (S. 128-146). London: Routledge. https://doi.org/10.4324/ 9781315167237.

Davis, F. D., \& Venkatesh, V. (1996). A critical assessment of potential measurement biases in the technology acceptance model: three experiments. International Journal of Human-Computer Studies, 45(1), 19-45. https://doi.org/10.1006/ijhc.1996.0040.

Davis, M.C., Challenger, R., Jayewardene, D.N., \& Clegg, C.W. (2014). Advancing socio-technical systems thinking: a call for bravery. Applied ergonomics, 45(2), 171-180. https://doi.org/10. 1016/j.apergo.2013.02.009.

Demerouti, E., Bakker, A.B., Nachreiner, F., \& Schaufeli, W.B. (2001). The job demands-resources model of burnout. Journal of Applied Psychology, 86, 499-512. https://doi.org/10.1037/ 0021-9010.86.3.499.

Demirkan, H., \& Spohrer, J. (2015). T-shaped innovators: identifying the right talent to support service innovation. ResearchTechnology Management, 58(5), 12-15. https://doi.org/10.5437/ 08956308 X5805007.

Earley, P. C., Northcraft, G. B., Lee, C., \& Lituchy, T. R. (1990). Impact of process and outcome feedback on the relation of goal setting to task performance. Academy of Management Journal, 33, 87-105. https://doi.org/10.5465/256353.

Eccles, R. G. (1981). The quasifirm in the construction industry. Journal of Economic Behavior and Organization, 2(4), 335-357. https://doi.org/10.1016/0167-2681(81)90013-5.

Espinosa, J. A., Slaughter, S. A., Kraut, R. E., \& Herbsleb, J.D. (2007). Familiarity, complexity, and team performance in geographically distributed software development. Organization Science, 18, 613-630. https://doi.org/10.1287/orsc.1070.0297.

Ferreira, A., Antunes, P., \& Herskovic, V. (2011). Improving group attention: an experiment with synchronous brainstorming. Group Decision and Negotiation, 20, 643-666. https://doi.org/10.1007/ s10726-011-9233-y.

Fiedler, F.E. (1967). A theory of leadership effectiveness. New York: McGraw-Hill.

Fischer, M., Ashcraft, H. W., Reed, D., \& Khanzode, A. (2017). Integrating project delivery. A simple framework for putting IPD into action. Hoboken: John Wiley \& Sons. https://doi.org/10.1002/ 9781119179009

Forgues, E.C., Carignan, V., Forgues, D., \& Rajeb, S.B. (2016). A framework for improving collaboration patterns in BIM projects. In International conference on cooperative design, visualization and engineering (S. 34-42). Cham: Springer. https://doi.org/10. 1007/978-3-319-46771-9_5.

Friese, M. (1998). Kooperation als Wettbewerbsstrategie für Dienstleistungsunternehmen. Wiesbaden: Gabler. https://doi.org/10. 1007/978-3-663-01487-4.

Geister, S., Konradt, U., \& Hertel, G. (2006). Effects of process feedback on motivation, satisfaction, and performance in virtual teams. Small Group Research, 37, 459-489. https://doi.org/10. 1177/1046496406292337.

Handke, L., \& Kauffeld, S. (2019). Alles eine Frage der Zeit? Herausforderungen virtueller Teams und deren Bewältigung am Beispiel der Softwareentwicklung. Gruppe. Interaktion. Organisation. Zeitschrift für Angewandte Organisationspsychologie (GIO), 50(1), 33-41. https://doi.org/10.1007/s11612-019-00445-5.

Handke, L., Klonek, F.E., Parker, S. K., \& Kauffeld, S. (2020). Interactive effects of team virtuality and work design on team functioning. Small Group Research, 51(1), 3-47. https://doi.org/10.1177/ 1046496419863490.

Handke, L., Schulte, E.-M., Schneider, K., \& Kauffeld, S. (2019). Teams, time, and technology: variations of media use over project phases. Small Group Research, 50(2), 266-305. https://doi.org/ 10.1177/1046496418824151.

Hansen, M. T., \& von Oetinger, B. (2001). Introducing T-shaped managers. Knowledge management's next generation. Harvard business review, 79(3), 106-116.

Hertel, G., Geister, S., \& Konradt, U. (2005). Managing virtual teams: a review of current empirical research. Human Resource Management Review, 15, 69-95. https://doi.org/10.1016/j.hrmr.2005.01. 002.

Hertel, G., Konradt, U., \& Orlikowski, B. (2004). Managing distance by interdependence: Goal setting, task interdependence, and team-based rewards in virtual teams. European Journal of Work and Organizational Psychology, 13, 1-28. https://doi.org/ 10.1080/13594320344000228.

Huang, W. W., \& Wei, K. K. (2000). An empirical investigation of the effects of group support systems (GSS) and task type on group interactions from an influence perspective. Journal of Management Information Systems, 17, 181-206. https://doi.org/10.1080/ 07421222.2000 .11045647

Jackson, P. R., Wall, T. D., Martin, R., \& Davids, K. (1993). New measures of job control, cognitive demand, and production responsibility. Journal of Applied Psychology, 78, 753-762. https://doi. org/10.1037/0021-9010.78.5.753.

Jacobsson, M., \& Merschbrock, C. (2018). BIM coordinators: a review. Engineering, Construction and Architectural Management, 25, 989-1008. https://doi.org/10.1108/ECAM-03-2017-0050.

Jasperson, J.S., Carter, P.E., \& Zmud, R.W. (2005). A comprehensive conceptualization of post-adoptive behaviors associated with information technology enabled work systems. MIS quarterly, 29(3), 525-557.

Jones, C., \& Lichtenstein, B. B. (2009). Temporary inter-organizational projects: how temporal and social embeddedness enhance coordination and manage uncertainty. In S. Cropper, C. Huxham, M. Ebers \& P.S. Ring (Hrsg.), The oxford handbook of interorganizational relations (S. 231-255). Oxford: Oxford University Press. https://doi.org/10.1093/oxfordhb/9780199282944.003. 0009.

Kahai, S. S., Sosik, J. J., \& Avolio, B. J. (2003). Effects of leadership style, anonymity, and rewards on creativity-relevant processes and outcomes in an electronic meeting system context. The Leadership Quarterly, 14, 499-524. https://doi.org/10.1016/S10489843(03)00049-3.

Kahn, R.L., Wolfe, D.M., Quinn, R.P., Snoek, J.D., \& Rosenthal, R. A. (1964). Occupational stress: studies in role conflict and ambiguity. New York: John Wiley.

Kauffeld, S., Handke, L., \& Straube, J. (2016). Verteilt und doch verbunden: Virtuelle Teamarbeit. Gruppe. Interaktion. Organisati- 
on. Zeitschrift für angewandte Organisationspsychologie, 47(1), 43-51. https://doi.org/10.1007/s11612-016-0308-8.

Kim, Y., Hiltz, S. R., \& Turoff, M. (2002). Coordination structures and system restrictiveness in distributed group support systems. Group Decision and Negotiation, 11, 379-404. https://doi.org/10. 1023/A:1020492305910.

Klemt-Albert, K. (2018). Durch die Digitalisierung zu mehr Produktivität in der Bauwirtschaft. Bautechnik, 95(3), 187-188. https:// doi.org/10.1002/bate.201870303.

Kocijan, M. (2018). Digitalisierung im Bausektor. ifo Schnelldienst, $71(1), 42-45$

Kranzberg, M. (1986). Technology and history: "Kranzberg's laws". Technology and culture, 27(3), 544-560. https://doi.org/10.2307/ 3105385.

Laughlin, P. R., \& Ellis, A. L. (1986). Demonstrability and social combination processes on mathematical intellective tasks. Journal of Experimental Social Psychology, 22, 177-189. https://doi.org/10. 1016/0022-1031(86)90022-3.

Lichtblau, K., Schleiermacher, T., Goecke, H., \& Schützdeller, P. (2018). Digitalisierung der KMU in Deutschland. Köln: IW Consult.

Linderoth, H. (2010). Understanding adoption and use of BIM as the creation of actor networks. Automation in Construction, 19(1), 66-72. https://doi.org/10.1016/j.autcon.2009.09.003.

Loose, A. (2001). Kooperationsverbünde und Unternehmensnetzwerke im Bau- und Ausbauhandwerk - Auf dem Weg zum „virtuellen“ Unternehmen? In J. Howaldt, R. Kopp \& P. Flocken (Hrsg.), Kooperationsverbünde und regionale Modernisierung. Theorie und Praxis der Netzwerkarbeit (S. 143-157). Wiesbaden: Gabler. https://doi.org/10.1007/978-3-322-90831-5.

Malhotra, A., \& Majchrzak, A. (2014). Enhancing performance of geographically distributed teams through targeted use of information and communication technologies. Human Relations, 67, 389-411. https://doi.org/10.1177/0018726713495284.

Manning, R., \& Messner, J. (2008). Case studies in BIM implementation for programming of healthcare facilities. Electronic Journal of Information Technology in Construction, 13, 446-457.

Martínez-Moreno, E., Zornoza, A., Orengo, V., \& Thompson, L.F. (2015). The effects of team self-guided training on conflict management in virtual teams. Group Decision and Negotiation, 24, 905-923. https://doi.org/10.1007/s10726-014-9421-7.

Maznevski, M.L., \& Chudoba, K.M. (2000). Bridging space over time: global virtual team dynamics and effectiveness. Organization Science, 11, 473-492. https://doi.org/10.1287/orsc.11.5.473. 15200.

McGrath, J.E. (1984). Groups: interaction and performance. Englewood Cliffs: Prentice Hall.

Miettinen, R., \& Paavola, S. (2014). Beyond the BIM utopia: approaches to the development and implementation of building information modeling. Automation in construction, 43, 84-91. https://doi. org/10.1016/j.autcon.2014.03.009.

Morgeson, F.P., \& Humphrey, S.E. (2006). The Work Design Questionnaire (WDQ): developing and validating a comprehensive measure for assessing job design and the nature of work. Journal of Applied Psychology, 91, 1321-1339. https://doi.org/10.1037/ 0021-9010.91.6.1321.

Morgeson, F.P., \& Humphrey, S.E. (2008). Job and team design: toward a more integrative conceptualization of work design. In J.J. Martocchio (Hrsg.), Research in personnel and human resources management (Bd. 27, S. 39-91). London: Emerald. https://doi.org/10.1016/s0742-7301(08)27002-7.

Naegele, L., Kortsch, T., Paulsen, H., Wiemers, D., Kauffeld, S., \& Frerichs, F. (2015). Zukunft im Blick: Trends erkennen, Kompetenzen entwickeln, Chancen nutzen. Drei Perspektiven auf die Zukunft des Handwerks. Braunschweig: Technische Universität Braunschweig. Projektbericht „Integrierte Kompetenzentwicklung im Handwerk"
Neff, G., Fiore-Silfvast, B., \& Dossick, C. S. (2010). A case study of the failure of digital communication to cross knowledge boundaries in virtual construction. Information, Communication \& Society, 13, 556-573. https://doi.org/10.1080/13691181003645970.

Ortiz De Guinea, A., Webster, J., \& Staples, D. S. (2012). A meta-analysis of the consequences of virtualness on team functioning. Information \& Management, 49, 301-308. https://doi.org/10.1016/ j.im.2012.08.003.

Painter, G., Posey, P., Austrom, D., Tenkasi, R., Barrett, B., \& Merck, B. (2016). Sociotechnical systems design: coordination of virtual teamwork in innovation. Team Performance Management, 22, 354-369. https://doi.org/10.1108/TPM-12-2015-0060.

Parker, S., \& Grote, G. (2020). Automation, algorithms, and beyond: why work design matters more than ever in a digital world. Applied Psychology. https://doi.org/10.1111/apps.12241.

Parker, S. K., Van den Broeck, A., \& Holman, D. (2017). Work design influences: a synthesis of multilevel factors that affect the design of jobs. Academy of Management Annals, 11(1), 267-308. https:// doi.org/10.5465/annals.2014.0054.

Schlicher, K. D., Paruzel, A., Steinmann, B., \& Maier, G. W. (2018). Change Management für die Einführung digitaler Arbeitswelten. In G. Maier, G. Engels \& E. Steffen (Hrsg.), Handbuch Gestaltung digitaler und vernetzter Arbeitswelten (S. 1-36). Berlin, Heidelberg, New York: Springer.

Schreiber, M., \& Engelmann, T. (2010). Knowledge and information awareness for initiating transactive memory system processes of computer-supported collaborating ad hoc groups. Computers in Human Behavior, 26, 1701-1709. https://doi.org/10.1016/j.chb. 2010.06.019.

Sebastian, R. (2011). Changing roles of the clients, architects and contractors through BIM. Engineering, Construction and Architectural Management, 18(2), 176-187. https://doi.org/10.1108/ 09699981111111148.

Statistisches Bundesamt (2019). Ausgewählte Zahlen für die Bauwirtschaft. Wiesbaden: DeStatis. retrieved from https://www.destatis. de/DE/Themen/Branchen-Unternehmen/Bauen/Publikationen/ Downloads-Querschnitt/bauwirtschaft-1020210191054.pdf?_ blob=publicationFile

Sträter, O., \& Bengler, K. (2019). Positionspapier Digitalisierung der Arbeitswelt. Zeitschrift für Arbeitswissenschaften, 73, 243-245. https://doi.org/10.1007/s41449-019-00161-2.

Thomas, P. (2016). Branchenanalyse SHK-Handwerk: Aktuelle Herausforderungen und Chancen. Working Paper Forschungsförderung, No. 010. Düsseldorf: Hans- Böckler-Stiftung. http://nbnresolving.de/urn:nbn:de:101:1-201606092019

Thompson, J. D. (1967). Organizations in action. New York: McGrawHill.

Ulich, E. (2013). Arbeitssysteme als soziotechnische Systeme - eine Erinnerung. Journal Psychologie des Alltagshandelns, 6(1), 4-12.

Venkatesh, V., \& Bala, H. (2008). Technology acceptance model 3 and a research agenda on interventions. Decision Sciences, 39(2), 273-315. https://doi.org/10.1111/j.1540-5915.2008.00192.x.

Wall, T.D., Corbett, J.M., Martin, R., Clegg, C.W., \& Jackson, P.R. (1990). Advanced manufacturing technology, work design, and performance: a change study. Journal of Applied Psychology, 75, 691-697. https://doi.org/10.1037/0021-9010.75.6.691.

Wollny, V., \& Paul, H. (2015). Die SWOT-Analyse: Herausforderungen der Nutzung in den Sozialwissenschaften. In M. Niederberger \& S. Wassermann (Hrsg.), Methoden der Experten- und Stakeholdereinbindung in der sozialwissenschaftlichen Forschung (S. 189-213). Wiesbaden: Springer VS. https://doi.org/10.1007/ 978-3-658-01687-6_10.

Wu, W., Mayo, G., McCuen, T.L., Issa, R.R., \& Smith, D. K. (2018). Building information modeling body of knowledge. I: Background, framework, and initial development. Journal of Construction Engineering and Management, 144(8), 1-9. https://doi. org/10.1061/(ASCE)CO.1943-7862.0001518. 


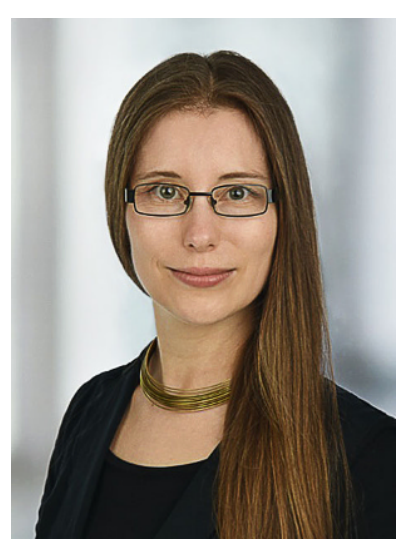

Dr. Sandra Rothenbusch ist wissenschaftliche Mitarbeiterin am Lehrstuhl für Arbeits-, Organisations- und Sozialpsychologie der Technischen Universität Braunschweig. Sie promovierte im Bereich der Begabungsforschung und beschäftigt sich seitdem mit der Kompetenzentwicklung und $\mathrm{Ar}$ beitsgestaltung in einer digitalisierten Arbeitswelt.

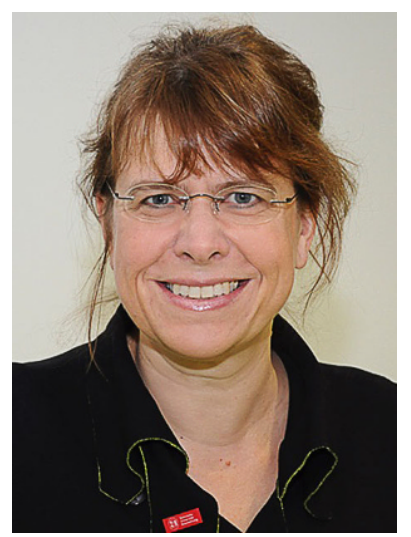

Prof. Dr. Simone Kauffeld ist Inhaberin des Lehrstuhls für Arbeit-, Organisations- und Sozialpsychologie der Technischen Universität Braunschweig. In ihrer Forschungstätigkeit setzt sie sich in zahlreichen Projekten mit den Themen Kompetenzentwicklung und -management (Training und Transfer), Team und Führung, Karriere/Coaching sowie Veränderungen in Organisation und Arbeit auseinander. Das Thema Digitalisierung ist als Querschnittsthema präsent. Als Herausgeberin hat sie die Zeitschriften „PersonalQUARTERLY“ und „Gruppe. Interaktion. Organisation“ neu aufgesetzt und gibt Buchreihen zur Arbeits- und Organisationspsychologie heraus. Um aktiven Wissenstransfer zu leisten, hat sie 2008 die 4A-SIDE GmbH gegründet, die psychologische Expertise mit IT-Kompetenz verbindet. 